\title{
The Laplace Transform of the Cut-and-Join Equation and the Bouchard-Mariño Conjecture on Hurwitz Numbers
}

\author{
by \\ Bertrand Eynard, Motohico Mulase, and Bradley SAFnuK
}

\begin{abstract}
We calculate the Laplace transform of the cut-and-join equation of Goulden, Jackson and Vakil. The result is a polynomial equation that has the topological structure identical to the Mirzakhani recursion formula for the Weil-Petersson volume of the moduli space of bordered hyperbolic surfaces. We find that the direct image of this Laplace transformed equation via the inverse of the Lambert $\mathrm{W}$-function is the topological recursion formula for Hurwitz numbers conjectured by Bouchard and Mariño using topological string theory.

2010 Mathematics Subject Classification: 14H10, 14N10, 14N35, 05A15, 05A17, 81T45. Keywords: Hurwitz numbers, cut-and-join equation, linear Hodge integral, topological recursion, Lambert curve.
\end{abstract}

\section{Contents}

\begin{tabular}{l}
\begin{tabular}{|ll|l|}
\hline 1 & Introduction & 630 \\
\hline 2 & The Laplace transform of the ELSV formula & 635 \\
\hline 3 & The cut-and-join equation and its Laplace transform & 640 \\
\hline 4 & The Bouchard-Mariño recursion formula for Hurwitz numbers & 646 \\
\hline 5 & Residue calculation 651 \\
\hline 6 & Analysis of the Laplace transforms on the Lambert curve & \\
\hline 7 & Proof of the Bouchard-Mariño topological recursion formula & \\
\hline References 668 & 663
\end{tabular} \\
\hline
\end{tabular}

This is a contribution to the special issue "The golden jubilee of algebraic analysis".

Communicated by M. Kashiwara. Received December 15, 2009.

B. Eynard: Service de Physique Théorique de Saclay, Gif-sur-Yvette, F-91191 Cedex, France; e-mail: bertrand.eynard@cea.fr

M. Mulase: Department of Mathematics, University of California, Davis, CA 95616-8633, USA; e-mail: mulase@math.ucdavis.edu

B. Safnuk: Department of Mathematics, Central Michigan University,

Mount Pleasant, MI 48859, USA;

e-mail: brad.safnuk@cmich.edu

(C) 2011 Research Institute for Mathematical Sciences, Kyoto University. All rights reserved. 


\section{$\S 1$. Introduction}

The purpose of this paper is to give a proof of the Bouchard-Mariño conjecture 3] on Hurwitz numbers using the Laplace transform of the celebrated cut-andjoin equation of Goulden, Jackson, and Vakil [17, 43. The cut-and-join equation, which seems to have been essentially known to Hurwitz [23, expresses the Hurwitz number of a given genus and profile (partition) in terms of those corresponding to profiles modified by either cutting a part into two pieces or joining two parts into one. This equation holds for an arbitrary partition $\mu$. We calculate the Laplace transform of this equation with $\mu$ as the summation variable. The result is a polynomial equation 38.

A Hurwitz cover is a holomorphic mapping $f: X \rightarrow \mathbb{P}^{1}$ from a connected nonsingular projective algebraic curve $X$ of genus $g$ to the projective line $\mathbb{P}^{1}$ with only simple ramifications except for $\infty \in \mathbb{P}^{1}$. Such a cover is further refined by specifying its profile, which is a partition $\mu=\left(\mu_{1} \geq \cdots \geq \mu_{\ell}>0\right)$ of the degree of the covering $\operatorname{deg} f=|\mu|=\mu_{1}+\cdots+\mu_{\ell}$. The length $\ell(\mu)=\ell$ of this partition is the number of points in the inverse image $f^{-1}(\infty)=\left\{p_{1}, \ldots, p_{\ell}\right\}$ of $\infty$. Each part $\mu_{i}$ gives a local description of the map $f$, which is given by $z \mapsto z^{\mu_{i}}$ in terms of a local coordinate $z$ of $X$ around $p_{i}$. The number $h_{g, \mu}$ of topological types of Hurwitz covers of given genus $g$ and profile $\mu$, counted with the weight factor $1 / \mid$ Aut $f \mid$, is the Hurwitz number we shall deal with in this paper. A remarkable formula due to Ekedahl, Lando, Shapiro and Vainshtein 8, 21, 41, relates Hurwitz numbers and Gromov-Witten invariants. For genus $g \geq 0$ and a partition $\mu$ subject to the stability condition $2 g-2+\ell(\mu)>0$, the ELSV formula states that

$$
h_{g, \mu}=\frac{(2 g-2+\ell(\mu)+|\mu|) !}{|\operatorname{Aut}(\mu)|} \prod_{i=1}^{\ell(\mu)} \frac{\mu_{i}^{\mu_{i}}}{\mu_{i} !} \int_{\overline{\mathcal{M}}_{g, \ell(\mu)}} \frac{\Lambda_{g}^{\vee}(1)}{\prod_{i=1}^{\ell(\mu)}\left(1-\mu_{i} \psi_{i}\right)},
$$

where $\overline{\mathcal{M}}_{g, \ell}$ is the Deligne-Mumford moduli stack of stable algebraic curves of genus $g$ with $\ell$ distinct marked points, $\Lambda_{g}^{\vee}(1)=1-c_{1}(\mathbb{E})+\cdots+(-1)^{g} c_{g}(\mathbb{E})$ is the alternating sum of the Chern classes of the Hodge bundle $\mathbb{E}$ on $\overline{\mathcal{M}}_{g, \ell}, \psi_{i}$ is the $i$-th tautological cotangent class, and $\operatorname{Aut}(\mu)$ denotes the group of permutations of equal parts of the partition $\mu$. The linear Hodge integrals are the rational numbers defined by

$$
\left\langle\tau_{n_{1}} \cdots \tau_{n_{\ell}} c_{j}(\mathbb{E})\right\rangle=\int_{\overline{\mathcal{M}}_{g, \ell}} \psi_{1}^{n_{1}} \cdots \psi_{\ell}^{n_{\ell}} c_{j}(\mathbb{E}),
$$

which is 0 unless $n_{1}+\cdots+n_{\ell}+j=3 g-3+\ell$. To present our main theorem, let us introduce a series of polynomials $\hat{\xi}_{n}(t)$ of degree $2 n+1$ in $t$ for $n \geq 0$ by the recursion formula

$$
\hat{\xi}_{n}(t)=t^{2}(t-1) \frac{d}{d t} \hat{\xi}_{n-1}(t)
$$


with the initial condition $\hat{\xi}_{0}(t)=t-1$. This differential operator appears in [19]. The Laplace transform of the cut-and-join equation gives the following formula.

Theorem 1.1 ([38]). Linear Hodge integrals satisfy recursion relations given as a series of equations of symmetric polynomials in $\ell$ variables $t_{1}, \ldots, t_{\ell}$ :

$$
\begin{aligned}
& \sum_{n_{L}}\left\langle\tau_{n_{L}} \Lambda_{g}^{\vee}(1)\right\rangle_{g, \ell}\left((2 g-2+\ell) \hat{\xi}_{n_{L}}\left(t_{L}\right)+\sum_{i=1}^{\ell} \frac{1}{t_{i}} \hat{\xi}_{n_{i}+1}\left(t_{i}\right) \hat{\xi}_{L \backslash\{i\}}\left(t_{L \backslash\{i\}}\right)\right) \\
& =\sum_{i<j} \sum_{m, n_{L \backslash\{i, j\}}}\left\langle\tau_{m} \tau_{n_{L \backslash\{i, j\}}} \Lambda_{g}^{\vee}(1)\right\rangle_{g, \ell-1} \hat{\xi}_{n_{L \backslash\{i, j\}}}\left(t_{L \backslash\{i, j\}}\right) \\
& \times \frac{\hat{\xi}_{m+1}\left(t_{i}\right) \hat{\xi}_{0}\left(t_{j}\right) t_{i}^{2}-\hat{\xi}_{m+1}\left(t_{j}\right) \hat{\xi}_{0}\left(t_{i}\right) t_{j}^{2}}{t_{i}-t_{j}} \\
& +\frac{1}{2} \sum_{i=1}^{\ell} \sum_{n_{L \backslash\{i\}}} \sum_{a, b}\left(\left\langle\tau_{a} \tau_{b} \tau_{n_{L \backslash\{i\}}} \Lambda_{g-1}^{\vee}(1)\right\rangle_{g-1, \ell+1}\right. \\
& \left.+\sum_{\substack{g_{1}+g_{2}=g \\
I \sqcup J=L \backslash\{i\}}}^{\text {stable }}\left\langle\tau_{a} \tau_{n_{I}} \Lambda_{g_{1}}^{\vee}(1)\right\rangle_{g_{1},|I|+1}\left\langle\tau_{b} \tau_{n_{J}} \Lambda_{g_{2}}^{\vee}(1)\right\rangle_{g_{2},|J|+1}\right) \\
& \times \hat{\xi}_{a+1}\left(t_{i}\right) \hat{\xi}_{b+1}\left(t_{i}\right) \hat{\xi}_{n_{L \backslash\{i\}}}\left(t_{L \backslash\{i\}}\right),
\end{aligned}
$$

where $L=\{1, \ldots, \ell\}$ is an index set, and for a subset $I \subset L$, we denote

$$
t_{I}=\left(t_{i}\right)_{i \in I}, \quad n_{I}=\left\{n_{i} \mid i \in I\right\}, \quad \tau_{n_{I}}=\prod_{i \in I} \tau_{n_{i}}, \quad \hat{\xi}_{n_{I}}\left(t_{I}\right)=\prod_{i \in I} \hat{\xi}_{n_{i}}\left(t_{i}\right) .
$$

The last summation in the formula is taken over all partitions of $g$ and decompositions of $L \backslash\{i\}$ into disjoint subsets $I \sqcup J=L \backslash\{i\}$ subject to the stability condition $2 g_{1}-1+|I|>0$ and $2 g_{2}-1+|J|>0$.

Remark 1.2. We note a similarity of the above formula and the Mirzakhani recursion formula for the Weil-Petersson volume of the moduli space of bordered hyperbolic surfaces of genus $g$ with $\ell$ closed geodesic boundaries [35, 36].

(1) There is no a priori reason for the Laplace transform to be a polynomial equation.

(2) The above formula is a topological recursion. For an algebraic curve of genus $g \geq 0$ and $\ell \geq 1$ distinct marked points on it, the absolute value of the Euler characteristic of the $\ell$-punctured Riemann surface, $2 g-2+\ell$, defines a complexity of the moduli space $\overline{\mathcal{M}}_{g, \ell} \cdot 1.2$ gives an effective method of calculating the linear Hodge integrals of complexity $n>0$ from those with complexity $n-1$. 
(3) When we restrict 1.2 to the homogeneous highest degree terms, the equation reduces to the Witten-Kontsevich theorem of $\psi$-class intersections [7, 27, 44].

Let us explain the background of our work. Independent of the recent geometric and combinatorial works [17, 35, 36, 43], a theory of topological recursions has been developed in the matrix model/random matrix theory community [9, 13]. Its culmination is the topological recursion formula established in [13. There are three ingredients in this theory: the Cauchy differentiation kernel (which is referred to as the "Bergman kernel" in [3, 13]) of an analytic curve $C \subset \mathbb{C}^{2}$ in the $x y$-plane called a spectral curve, the standard holomorphic symplectic structure on $\mathbb{C}^{2}$, and the ramification behavior of the projection $\pi: C \rightarrow \mathbb{C}$ of the spectral curve to the $x$-axis. When $C$ is hyperelliptic whose ramification points are all real, the topological recursion solves 1-Hermitian matrix models for the potential function that determines the spectral curve. This means that the formula recursively computes all $n$-point correlation functions of the resolvent of random Hermitian matrices of an arbitrary size. By choosing a particular spectral curve of genus 0, the topological recursion [10, 13, 14 recovers the Virasoro constraint conditions for the $\psi$-class intersection numbers $\left\langle\tau_{n_{1}} \cdots \tau_{n_{\ell}}\right\rangle$ due to Witten [44] and Kontsevich [27, and the mixed intersection numbers $\left\langle\kappa_{1}^{m_{1}} \kappa_{2}^{m_{2}} \cdots \tau_{n_{1}} \cdots \tau_{n_{\ell}}\right\rangle$ due to Mulase-Safnuk 37. and Liu-Xu 31. Based on the work by Mariño 33. and Bouchard, Klemm, Mariño and Pasquetti [2] on remodeling the B-model topological string theory on the mirror curve of a toric Calabi-Yau 3-fold, Bouchard and Mariño 3] conjecture that when one uses the Lambert curve

$$
C=\left\{(x, y) \mid x=y e^{-y}\right\} \subset \mathbb{C}^{*} \times \mathbb{C}^{*}
$$

as the spectral curve, the topological recursion formula of Eynard and Orantin should compute the generating functions

$$
\begin{aligned}
H_{g, \ell}\left(x_{1}, \ldots, x_{\ell}\right) & =\sum_{\mu: \ell(\mu)=\ell} \frac{\mu_{1} \cdots \mu_{\ell}}{(2 g-2+\ell+|\mu|) !} h_{g, \mu} \sum_{\sigma \in S_{\ell}} \prod_{i=1}^{\ell} x_{\sigma(i)}^{\mu_{i}-1} \\
= & \sum_{n_{1}+\cdots+n_{\ell} \leq 3 g-3+\ell}\left\langle\tau_{n_{1}} \cdots \tau_{n_{\ell}} \Lambda_{g}^{\vee}(1)\right\rangle \prod_{i=1}^{\ell} \sum_{\mu_{i}=1}^{\infty} \frac{\mu_{i}^{\mu_{i}+1+n_{i}}}{\mu_{i} !} x_{i}^{\mu_{i}-1}
\end{aligned}
$$

of Hurwitz numbers for all $g \geq 0$ and $\ell>0$. Here the sum in the first line is taken over all partitions $\mu$ of length $\ell$, and $S_{\ell}$ is the symmetric group of $\ell$ letters.

Our discovery of this paper is that the Laplace transform of the combinatorics, the cut-and-join equation in our case, explains the role of the Lambert curve, the ramification behavior of the projection $\pi: C \rightarrow \mathbb{C}^{*}$, the Cauchy differentiation kernel on $C$, and residue calculations that appear in the theory of 


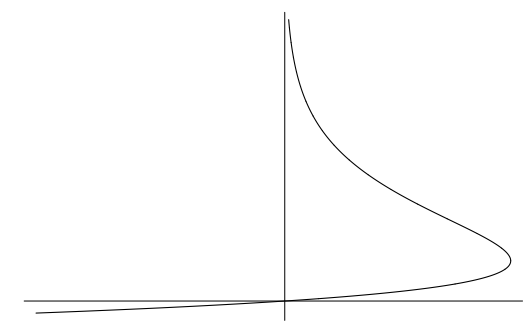

Figure 1.1. The Lambert curve $C \subset \mathbb{C}^{*} \times \mathbb{C}^{*}$ defined by $x=y e^{-y}$.

topological recursion. As a consequence of this explanation, we obtain a proof of the Bouchard-Mariño conjecture [3. For this purpose, it is essential to use a different parametrization of the Lambert curve:

$$
x=e^{-(w+1)} \quad \text { and } \quad y=\frac{t-1}{t} .
$$

The coordinate $w$ is the parameter of the Laplace transformation, which changes a function of positive integers to a complex analytic function of $w$. Recall the Stirling expansion

$$
e^{-k} \frac{k^{k+n}}{k !} \sim \frac{1}{\sqrt{2 \pi}} k^{n-1 / 2},
$$

which makes its Laplace transform a function of $\sqrt{w}$. Note that the $x$-projection $\pi$ of the Lambert curve (1.3) is locally a double-sheeted covering around its unique critical point $\left(x=e^{-1}, y=1\right)$. Therefore, the Laplace transform of the ELSV formula (1.1) naturally lives on the Lambert curve $C$ rather than on the $w$-coordinate plane. Note that $C$ is an analytic curve of genus 0 and $t$ is its global coordinate. The point at infinity $t=\infty$ is the ramification point of the projection $\pi$. In terms of these coordinates, the Laplace transform of the ELSV formula becomes a polynomial in $t$-variables.

The Bouchard-Mariño conjecture is proved as follows. A topological recursion of 13 is always given as a residue formula of symmetric differential forms on the spectral curve. The Laplace-transformed cut-and-join equation 1.2 is an equation among primitives of differential forms. We first take the exterior differential of this equation. We then analyze the role of the residue calculation in the theory of topological recursion [3, 13, and find that it is equivalent to evaluating the form at $q \in C$ and its conjugate point $\bar{q} \in C$ with respect to the local Galois covering $\pi: C \rightarrow \mathbb{C}$ near its critical point. This means all residue calculations are replaced by an algebraic operation of taking the direct image of the differential form via the projection $\pi$. We find that the direct image of $(1.2)$ then becomes identical to the conjectured formula $(1.5)$. 
Theorem 1.3 (The Bouchard-Mariño Conjecture). The linear Hodge integrals satisfy exactly the same topological recursion formula discovered in [13]:

$$
\begin{aligned}
& \sum_{n, n_{L}}\left\langle\tau_{n} \tau_{n_{L}} \Lambda_{g}^{\vee}(1)\right\rangle_{g, \ell+1} d \hat{\xi}_{n}(t) \otimes d \hat{\xi}_{n_{L}}\left(t_{L}\right) \\
= & \sum_{i=1}^{\ell} \sum_{m, n_{L \backslash\{i\}}}\left\langle\tau_{m} \tau_{n_{L \backslash\{i\}}} \Lambda_{g}^{\vee}(1)\right\rangle_{g, \ell} P_{m}\left(t, t_{i}\right) d t \otimes d t_{i} \otimes d \hat{\xi}_{n_{L} \backslash\{i\}}\left(t_{L \backslash\{i\}}\right) \\
+ & \left(\sum_{a, b, n_{L}}\left\langle\tau_{a} \tau_{b} \tau_{n_{L}} \Lambda_{g-1}^{\vee}(1)\right\rangle_{g-1, \ell+2}\right. \\
+ & \left.\sum_{\substack{g_{1}+g_{2}=g \\
I \sqcup J=L}}^{\text {stable }} \sum_{\substack{a, n_{I} \\
b, n_{J}}}\left\langle\tau_{a} \tau_{n_{I}} \Lambda_{g_{1}}^{\vee}(1)\right\rangle_{g_{1},|I|+1}\left\langle\tau_{b} \tau_{n_{J}} \Lambda_{g_{2}}^{\vee}(1)\right\rangle_{g_{2},|J|+1}\right) P_{a, b}(t) d t \otimes d \hat{\xi}_{n_{L}}\left(t_{L}\right),
\end{aligned}
$$

where

$$
d \hat{\xi}_{n_{I}}\left(t_{I}\right)=\bigotimes_{i \in I} \frac{d}{d t_{i}} \hat{\xi}_{n_{i}}\left(t_{i}\right) d t_{i} .
$$

The functions $P_{a, b}(t)$ and $P_{n}\left(t, t_{i}\right)$ are defined by taking the polynomial part of the expressions

$$
\begin{aligned}
& P_{a, b}(t) d t=\frac{1}{2}\left[\frac{t s(t)}{t-s(t)} \frac{d t}{t^{2}(t-1)}\left(\hat{\xi}_{a+1}(t) \hat{\xi}_{b+1}(s(t))+\hat{\xi}_{a+1}(s(t)) \hat{\xi}_{b+1}(t)\right)\right]_{+}, \\
& P_{n}\left(t, t_{i}\right) d t \otimes d t_{i}=d_{t_{i}}\left[\frac{t s(t)}{t-s(t)}\left(\frac{\hat{\xi}_{n+1}(t) d s(t)}{s(t)-t_{i}}+\frac{\hat{\xi}_{n+1}(s(t)) d t}{t-t_{i}}\right)\right]_{+},
\end{aligned}
$$

where $s(t)$ is the deck transformation of the projection $\pi: C \rightarrow \mathbb{C}^{*}$ around its critical point $\infty$.

The relation between the cut-and-join formula, $(1.2)$ and $\sqrt{1.5}$ is the following:

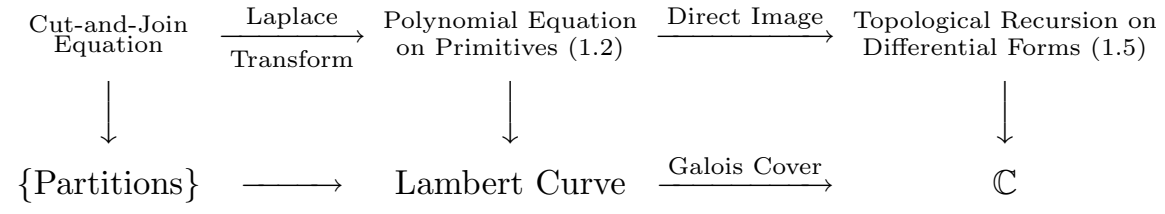

Mathematics of the topological recursion and its geometric realization includes still many more mysteries [2, 6, 13, 33. Among them is a relation to integrable systems such as the Kadomtsev-Petviashvili equations [13. In recent years these equations have played an essential role in the study of Hurwitz numbers 24, 25, 34, 40, 41, 42. Since the aim of this paper is to give a proof of the Bouchard-Mariño conjecture and to give a geometric interpretation of the topo- 
logical recursion for the Hurwitz case, we do not address this relation here. Since we relate the nature of the topological recursion and combinatorics by the Laplace transform, it is reasonable to ask: what is the inverse Laplace transform of the topological recursion in general? This question relates the Laplace transformation and mirror symmetry. These are interesting topics to be further explored.

It is possible to prove the Bouchard-Mariño formula without appealing to the cut-and-join equation. Indeed, a matrix integral expression of the generating function of Hurwitz numbers has been recently discovered in [1, and its spectral curve is identified as the Lambert curve. As a consequence, the symplectic invariant theory of matrix models [9, 11] is directly applicable to Hurwitz theory. The discovery of [1] is that the derivatives of the symplectic invariants of the Lambert curve give $H_{g, \ell}\left(x_{1}, \ldots, x_{\ell}\right)$ of 1.4$)$. The topological recursion formula of Bouchard and Mariño then automatically follows. A deeper understanding of the interplay between these two totally different techniques is desirable.

Although our statement is simple and clear, technical details are quite involved. We have decided to provide all key details in this paper, believing that some of the analysis may be useful for further study of more general topological recursions. This explains the length of the current paper in the sections dealing with complex analysis and Laplace transforms.

The paper is organized as follows. We start by identifying the generating function 1.4 as the Laplace transform of the ELSV formula (1.1) in Section 2. We then calculate the Laplace transform of the cut-and-join equation in Section 3 following [38, and present the key idea of the proof of Theorem 1.1. In Section 4 we give the statement of the Bouchard-Mariño conjecture [3. We calculate the residues appearing in the topological recursion formula in Section 5 for the case of Hurwitz generating functions. The topological recursion becomes the algebraic relation as presented in Theorem 1.3. In Section 6 we prove technical statements necessary for reducing 1.2 to 1.5 as a Galois average. The final Section 7 is devoted to proving the Bouchard-Mariño conjecture.

As an effective recursion, 1.2 and 1.5 calculate linear Hodge integrals, and hence Hurwitz numbers through the ELSV formula. A computation has been performed by Michael Reinhard, an undergraduate student of UC Berkeley. We reproduce some of his tables at the end of the paper.

\section{§2. The Laplace transform of the ELSV formula}

In this section we calculate the Laplace transform of the ELSV formula as a function of partitions $\mu$. The result is a symmetric polynomial on the Lambert curve 1.3 . 
A Hurwitz cover is a smooth morphism $f: X \rightarrow \mathbb{P}^{1}$ of a connected nonsingular projective algebraic curve $X$ of genus $g$ to $\mathbb{P}^{1}$ that has only simple ramifications except for the point at infinity $\infty \in \mathbb{P}^{1}$. Let $f^{-1}(\infty)=\left\{p_{1}, \ldots, p_{\ell}\right\}$. Then the induced morphism of the formal completion $\hat{f}: \hat{X}_{p_{i}} \rightarrow \hat{\mathbb{P}}_{\infty}^{1}$ is given by $z \mapsto z^{\mu_{i}}$ with a positive integer $\mu_{i}$ in terms of a formal parameter $z$ around $p_{i} \in X$. We rearrange the integers $\mu_{i}$ so that $\mu=\left(\mu_{1} \geq \cdots \geq \mu_{\ell}>0\right)$ is a partition of $\operatorname{deg} f=|\mu|=\mu_{1}+\cdots+\mu_{\ell}$ of length $\ell(\mu)=\ell$. We call $f$ a Hurwitz cover of genus $g$ and profile $\mu$. A holomorphic automorphism of a Hurwitz cover is an automorphism $\phi$ of $X$ that preserves $f$ :

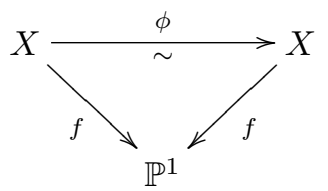

Two Hurwitz covers $f_{1}: X_{1} \rightarrow \mathbb{P}^{1}$ and $f_{2}: X_{2} \rightarrow \mathbb{P}^{1}$ are topologically equivalent if there is a homeomorphism $h: X_{1} \rightarrow X_{2}$ such that

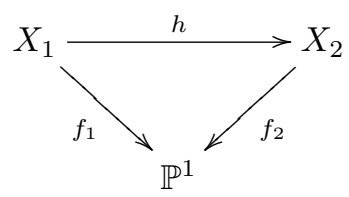

The Hurwitz number of type $(g, \mu)$ is defined by

$$
h_{g, \mu}=\sum_{[f]} \frac{1}{|\operatorname{Aut} f|},
$$

where the sum is taken over all topologically equivalent classes of Hurwitz covers of a given genus $g$ and profile $\mu$. Although $h_{g, \mu}$ appears to be a rational number, it is indeed an integer for most of the cases because $f$ has usually no nontrivial automorphisms. The celebrated ELSV formula [8, 21, 41] relates Hurwitz numbers and linear Hodge integrals on the Deligne-Mumford moduli stack $\overline{\mathcal{M}}_{g, \ell}$ consisting of stable algebraic curves of genus $g$ with $\ell$ distinct nonsingular marked points. Denote by $\pi_{g, \ell}: \overline{\mathcal{M}}_{g, \ell+1} \rightarrow \overline{\mathcal{M}}_{g, \ell}$ the natural projection and by $\omega_{\pi_{g, \ell}}$ the relative dualizing sheaf of the universal curve $\pi_{g, \ell}$. The Hodge bundle $\mathbb{E}$ on $\overline{\mathcal{M}}_{g, \ell}$ is defined by $\mathbb{E}=\left(\pi_{g, \ell}\right)_{*} \omega_{\pi_{g, \ell}}$, and the $\lambda$-classes are the Chern classes of the Hodge bundle:

$$
\lambda_{i}=c_{i}(\mathbb{E}) \in H^{2 i}\left(\overline{\mathcal{M}}_{g, \ell}, \mathbb{Q}\right) .
$$

Let $\sigma_{i}: \overline{\mathcal{M}}_{g, \ell} \rightarrow \overline{\mathcal{M}}_{g, \ell+1}$ be the $i$-th tautological section of $\pi$, and put $\mathcal{L}_{i}=$ $\sigma_{i}^{*}\left(\omega_{\pi_{g, \ell}}\right)$. The $\psi$-classes are defined by

$$
\psi_{i}=c_{1}\left(\mathcal{L}_{i}\right) \in H^{2}\left(\overline{\mathcal{M}}_{g, \ell}, \mathbb{Q}\right) .
$$


The ELSV formula then reads

$$
h_{g, \mu}=\frac{r !}{|\operatorname{Aut}(\mu)|} \prod_{i=1}^{\ell(\mu)} \frac{\mu_{i}^{\mu_{i}}}{\mu_{i} !} \int_{\overline{\mathcal{M}}_{g, \ell(\mu)}} \frac{\Lambda_{g}^{\vee}(1)}{\prod_{i=1}^{\ell(\mu)}\left(1-\mu_{i} \psi_{i}\right)},
$$

where $r=r(g, \mu)=2 g-2+\ell(\mu)+|\mu|$ is the number of simple ramification points of $f$.

The Deligne-Mumford stack $\overline{\mathcal{M}}_{g, \ell}$ is defined as the moduli space of stable curves satisfying the stability condition $2-2 g-\ell<0$. However, Hurwitz numbers are well defined for unstable geometries $(g, \ell)=(0,1)$ and $(0,2)$. It is an elementary exercise to show that

$$
h_{0, k}=k^{k-3} \quad \text { and } \quad h_{0,\left(\mu_{1}, \mu_{2}\right)}=\frac{\left(\mu_{1}+\mu_{2}\right) !}{\mu_{1}+\mu_{2}} \cdot \frac{\mu_{1}^{\mu_{1}}}{\mu_{1} !} \cdot \frac{\mu_{2}^{\mu_{2}}}{\mu_{2} !} .
$$

The ELSV formula remains true for unstable cases by defining

$$
\begin{aligned}
\int_{\overline{\mathcal{M}}_{0,1}} \frac{\Lambda_{0}^{\vee}(1)}{1-k \psi} & =\frac{1}{k^{2}}, \\
\int_{\overline{\mathcal{M}}_{0,2}} \frac{\Lambda_{0}^{\vee}(1)}{\left(1-\mu_{1} \psi_{1}\right)\left(1-\mu_{2} \psi_{2}\right)} & =\frac{1}{\mu_{1}+\mu_{2}} .
\end{aligned}
$$

Now fix an $\ell \geq 1$, and consider a partition $\mu$ of length $\ell$ as an $\ell$-dimensional vector

$$
\mu=\left(\mu_{1}, \ldots, \mu_{\ell}\right) \in \mathbb{N}^{\ell}
$$

consisting of positive integers. We define

$$
\begin{aligned}
H_{g}(\mu) & =\frac{|\operatorname{Aut}(\mu)|}{r(g, \mu) !} \cdot h_{g, \mu}=\prod_{i=1}^{\ell} \frac{\mu_{i}^{\mu_{i}}}{\mu_{i} !} \int_{\overline{\mathcal{M}}_{g, \ell}} \frac{\Lambda_{g}^{\vee}(1)}{\prod_{i=1}^{\ell}\left(1-\mu_{i} \psi_{i}\right)} \\
& =\sum_{n_{1}+\cdots+n_{\ell} \leq 3 g-3+\ell}\left\langle\tau_{n_{1}} \cdots \tau_{n_{\ell}} \Lambda_{g}^{\vee}(1)\right\rangle \prod_{i=1}^{\ell} \frac{\mu_{i}^{\mu_{i}+n_{i}}}{\mu_{i} !}
\end{aligned}
$$

as a function of $\mu$. Its Laplace transform

$$
\mathcal{H}_{g, \ell}(w)=\mathcal{H}_{g, \ell}\left(w_{1}, \ldots, w_{\ell}\right)=\sum_{\mu \in \mathbb{N}^{\ell}} H_{g}(\mu) e^{-\left(\mu_{1}\left(w_{1}+1\right)+\cdots+\mu_{\ell}\left(w_{\ell}+1\right)\right)}
$$

is the function we consider in this paper. We note that the automorphism group $\operatorname{Aut}(\mu)$ acts trivially on the function $e^{-\left(\mu_{1}\left(w_{1}+1\right)+\cdots+\mu_{\ell}\left(w_{\ell}+1\right)\right)}$, which explains its appearance in 2.3 . Since the coordinate change $x=e^{-(w+1)}$ identifies

$$
\frac{\partial^{\ell}}{\partial x_{1} \cdots \partial x_{\ell}} \mathcal{H}_{g, \ell}\left(w\left(x_{1}\right), \ldots, w\left(x_{\ell}\right)\right)=H_{g, \ell}\left(x_{1}, \ldots, x_{\ell}\right)
$$

the Laplace transform (2.4) is a primitive of the generating function (1.4). 
Before performing the exact calculation of the holomorphic function $\mathcal{H}_{g, \ell}(w)$, let us make a quick estimate here. From Stirling's formula

$$
e^{-k} \frac{k^{k+n}}{k !} \sim \frac{1}{\sqrt{2 \pi}} k^{n-1 / 2}
$$

it is obvious that $\mathcal{H}_{g, \ell}\left(w_{1}, \ldots, w_{\ell}\right)$ is holomorphic on $\operatorname{Re}\left(w_{i}\right)>0$ for all $i=1, \ldots, \ell$. Because of the half-integer powers of $\mu_{i}$ 's, the Laplace transform $\mathcal{H}_{g, \ell}(w)$ is expected to be a meromorphic function on a double-sheeted covering of the $w_{i}$-planes. Such a double covering is provided by the Lambert curve $C$ of $[1.3$. So we define

$$
t=1+\sum_{k=1}^{\infty} \frac{k^{k}}{k !} e^{-k(w+1)}
$$

which gives a global coordinate of $C$. The summation converges for $\operatorname{Re}(w)>0$, and the Lambert curve is expressed in terms of $w$ and $t$ coordinates as

$$
e^{-(w+1)}=\left(1-\frac{1}{t}\right) e^{-(1-1 / t)} .
$$

The $w$-projection $\pi: C \rightarrow \mathbb{C}$ is locally a double-sheeted covering at $t=\infty$. The inverse function of 2.6 is given by

$$
w=w(t)=-\frac{1}{t}-\log \left(1-\frac{1}{t}\right)=\sum_{m=2}^{\infty} \frac{1}{m} \frac{1}{t^{m}},
$$

which is holomorphic on $\operatorname{Re}(t)>1$. When considered as a functional equation, 2.8 has exactly two solutions: $t$ and

$$
s(t)=-t+\frac{2}{3}+\frac{4}{135} t^{-2}+\frac{8}{405} t^{-3}+\frac{8}{567} t^{-4}+\cdots
$$

This is the deck transformation of the projection $\pi: C \rightarrow \mathbb{C}$ near $t=\infty$ and satisfies the involution equation $s(s(t))=t$. It is analytic on $\mathbb{C} \backslash[0,1]$ and has logarithmic singularities at 0 and 1. Although $w(t)=w(s(t)), s(t)$ is not given by the Laplace transform (2.6).

Since the Laplace transform

$$
\hat{\xi}_{n}(t)=\sum_{k=1}^{\infty} \frac{k^{k+n}}{k !} e^{-k(w+1)}
$$

also naturally lives on $C$, it is a meromorphic function in $t$ rather than in $w$. Actually it is a polynomial of degree $2 n+1$ for $n \geq 0$ because of the recursion formula

$$
\hat{\xi}_{n+1}(t)=t^{2}(t-1) \frac{d}{d t} \hat{\xi}_{n}(t) \quad \text { for all } n \geq 0
$$


which follows from $2.6,2.10$, and 2.8 that implies

$$
-d w=\frac{d t}{t^{2}(t-1)}
$$

We note that the differential operator of 2.11) is discovered in [19]. For future convenience, we define

$$
\hat{\xi}_{-1}(t)=\frac{t-1}{t}=y
$$

which is indeed the $y$ coordinate of the original Lambert curve 1.3 . We now see that the Laplace transform

$$
\begin{aligned}
\widehat{\mathcal{H}}_{g, \ell}\left(t_{1}, \ldots, t_{\ell}\right) & =\mathcal{H}_{g, \ell}\left(w\left(t_{1}\right), \ldots, w\left(t_{\ell}\right)\right) \\
& =\sum_{\mu \in \mathbb{N}^{\ell}} H_{g}(\mu) e^{-\left(\mu_{1}\left(w_{1}+1\right)+\cdots+\mu_{\ell}\left(w_{\ell}+1\right)\right)} \\
& =\sum_{n_{1}+\cdots+n_{\ell} \leq 3 g-3+\ell}\left\langle\tau_{n_{1}} \cdots \tau_{n_{\ell}} \Lambda_{g}^{\vee}(1)\right\rangle \prod_{i=1}^{\ell} \hat{\xi}_{n_{i}}\left(t_{i}\right)
\end{aligned}
$$

is a symmetric polynomial in the $t$-variables when $2 g-2+\ell>0$.

It has been noted in [1, 10, 11, 13, 14] that the Airy curve $w=\frac{1}{2} v^{2}$ is a universal object of the topological recursion for the case of a genus 0 spectral curve with only one critical point. Analysis of the Airy curve provides a good control of the topological recursion formula for such cases. The Airy curve expression is also valid around any nondegenerate critical point of a general spectral curve. To switch to the local Airy curve coordinate, we define

$$
v=v(t)=t^{-1}+\frac{1}{3} t^{-2}+\frac{7}{36} t^{-3}+\frac{73}{540} t^{-4}+\frac{1331}{12960} t^{-5}+\cdots
$$

as a function of $t$ that solves

$$
\frac{1}{2} v^{2}=w=-\frac{1}{t}-\log \left(1-\frac{1}{t}\right)=-\frac{1}{s(t)}-\log \left(1-\frac{1}{s(t)}\right)=\sum_{m=2}^{\infty} \frac{1}{m} \frac{1}{t^{m}}
$$

Note that we are making a choice of the branch of the square root of $w$ that is consistent with 2.6. The involution 2.9 becomes simply

$$
v(t)=-v(s(t))
$$

The new coordinate $v$ plays a key role later when we reduce the Laplace transform of the cut-and-join equation 1.2 to the Bouchard-Mariño topological recursion (1.5). 


\section{$\S 3$. The cut-and-join equation and its Laplace transform}

In the modern times the cut-and-join equation for Hurwitz numbers was discovered in [17, 43, though it seems to have been known to Hurwitz 23. It has become an effective tool for studying the algebraic geometry of Hurwitz numbers and many related subjects $4,18,19,20,25,26,28,30,41,45$. In this section we calculate the Laplace transform of the cut-and-join equation following 38 .

The simplest way of presenting the cut-and-join equation is to use a different primitive of the same generating function of Hurwitz numbers (1.4). Let

$$
\mathbf{H}(s, \mathbf{p})=\sum_{g \geq 0} \sum_{\ell \geq 1} \mathbf{H}_{g, \ell}(s, \mathbf{p}), \quad \mathbf{H}_{g, \ell}(s, \mathbf{p})=\sum_{\mu: \ell(\mu)=\ell} h_{g, \mu} \mathbf{p}_{\mu} \frac{s^{r}}{r !},
$$

where $\mathbf{p}_{\mu}=p_{\mu_{1}} \cdots p_{\mu_{\ell}}$, and $r=2 g-2+\ell+|\mu|$ is the number of simple ramification points on $\mathbb{P}^{1}$. The summation is over all partitions of length $\ell$. Here $p_{k}$ is the powersum symmetric function

$$
p_{k}=\sum_{i \geq 1} x_{i}^{k}
$$

which is related to the monomial symmetric functions by

$$
\frac{\partial^{\ell}}{\partial x_{1} \cdots \partial x_{\ell}} \mathbf{p}_{\mu}=\sum_{\sigma \in S_{\ell}} \prod_{i=1}^{\ell} \mu_{i} x_{\sigma(i)}^{\mu_{i}-1}
$$

Therefore, we have

$$
\begin{aligned}
\frac{\partial^{\ell}}{\partial x_{1} \cdots \partial x_{\ell}} \mathbf{H}_{g, \ell}(1, \mathbf{p}) & =H_{g, \ell}\left(x_{1}, \ldots, x_{\ell}\right) \\
& =\sum_{\mu: \ell(\mu)=\ell} \frac{\mu_{1} \cdots \mu_{\ell}}{(2 g-2+\ell+|\mu|) !} h_{g, \mu} \sum_{\sigma \in S_{\ell}} \prod_{i=1}^{\ell} x_{\sigma(i)}^{\mu_{i}-1},
\end{aligned}
$$

which is the generating function of (1.4). Because of the identification 2.5), the primitives $\mathbf{H}_{g, \ell}(1, \mathbf{p})$ and $\widehat{\mathcal{H}}_{g, \ell}\left(t_{1}, \ldots, t_{\ell}\right)$ of 2.14 are essentially the same function, differing only by a constant.

Remark 3.1. Although we do not use this fact here, we note that $\mathbf{H}(s, \mathbf{p})$ is a one-parameter family of $\tau$-functions of the KP equations with $\frac{1}{k} p_{k}$ as the KP time variables [25, 40]. The parameter $s$ is the deformation parameter.

Let $z \in \mathbb{P}^{1}$ be a point at which the covering $f: X \rightarrow \mathbb{P}^{1}$ is simply ramified. Locally we can name sheets, so we assume sheets $a$ and $b$ are ramified over $z$. When we merge $z$ to $\infty$, one of the two things can happen: 
(1) The cut case. If both sheets are ramified at the same point $x_{i}$ of the inverse image $f^{-1}(\infty)=\left\{x_{1}, \ldots, x_{\ell}\right\}$, then the resulting ramification after merging $z$ to $\infty$ has a profile

$$
\left(\mu_{1}, \ldots, \widehat{\mu_{i}}, \ldots, \mu_{\ell}, \alpha, \mu_{i}-\alpha\right)=\left(\mu(\hat{i}), \alpha, \mu_{i}-\alpha\right)
$$

for $1 \leq \alpha<\mu_{i}$.

(2) Otherwise we are in the join case. If sheets $a$ and $b$ are ramified at two distinct points, say $x_{i}$ and $x_{j}$ above $\infty$, then the result of merging creates a new profile

$$
\left(\mu_{1}, \ldots, \widehat{\mu_{i}}, \ldots, \widehat{\mu_{j}}, \ldots, \mu_{\ell}, \mu_{i}+\mu_{j}\right)=\left(\mu(\hat{i}, \hat{j}), \mu_{i}+\mu_{j}\right) .
$$

Here the ${ }^{\wedge}$ sign means removing the entry. The above consideration tells us what happens to the generating function of the Hurwitz numbers when we differentiate it with respect to $s$, because it decreases the degree in $s$, or the number of simple ramification points, by 1 . Since the cut case may cause a disconnected covering, let us use the generating function of Hurwitz numbers allowing disconnected curves to cover $\mathbb{P}^{1}$. Then the cut-and-join equation takes the following simple form:

$$
\left[\frac{\partial}{\partial s}-\frac{1}{2} \sum_{\alpha, \beta \geq 1}\left((\alpha+\beta) p_{\alpha} p_{\beta} \frac{\partial}{\partial p_{\alpha+\beta}}+\alpha \beta p_{\alpha+\beta} \frac{\partial^{2}}{\partial p_{\alpha} \partial p_{\beta}}\right)\right] e^{\mathbf{H}(s, \mathbf{p})}=0 .
$$

It immediately implies

$$
\begin{aligned}
& \frac{\partial \mathbf{H}}{\partial s} \\
= & \frac{1}{2} \sum_{\alpha, \beta \geq 1}\left((\alpha+\beta) p_{\alpha} p_{\beta} \frac{\partial \mathbf{H}}{\partial p_{\alpha+\beta}}+\alpha \beta p_{\alpha+\beta} \frac{\partial^{2} \mathbf{H}}{\partial p_{\alpha} \partial p_{\beta}}+\alpha \beta p_{\alpha+\beta} \frac{\partial \mathbf{H}}{\partial p_{\alpha}} \cdot \frac{\partial \mathbf{H}}{\partial p_{\beta}}\right),
\end{aligned}
$$

which is the cut-and-join equation for the generating function $\mathbf{H}(s, \mathbf{p})$ of the number of connected Hurwitz coverings.

Let us now apply the ELSV formula (1.1) to (3.1). We obtain

$$
\begin{aligned}
\mathbf{H}_{g, \ell}(s, \mathbf{p}) & =\frac{1}{\ell !} \sum_{n_{L} \in \mathbb{N}^{\ell}}\left\langle\tau_{n_{L}} \Lambda_{g}^{\vee}(1)\right\rangle_{g, \ell} s^{2 g-2+\ell} \prod_{i=1}^{\ell} \sum_{\mu_{i}=1}^{\infty} \frac{\mu_{i}^{\mu_{i}+n_{i}}}{\mu_{i} !} s^{\mu_{i}} p_{\mu_{i}} \\
& =\frac{1}{\ell !} \sum_{\left(\mu_{1}, \ldots, \mu_{\ell}\right) \in \mathbb{N}^{\ell}} H_{g}(\mu) \mathbf{p}_{\mu} s^{r}=\sum_{\mu: \ell(\mu)=\ell} \frac{1}{|\operatorname{Aut}(\mu)|} H_{g}(\mu) \mathbf{p}_{\mu} s^{r}
\end{aligned}
$$

where $H_{g}(\mu)$ is introduced in 2.3. Now for every choice of $r \geq 1$ and a partition $\mu$, the coefficient of $\mathbf{p}_{\mu} s^{r-1}$ in the cut-and-join equation 3.3 gives 
Theorem 3.2 ([38]). The functions $H_{g}(\mu)$ of $(2.3)$ satisfy a recursion equation

$$
\begin{aligned}
& r(g, \mu) H_{g}(\mu)=\sum_{i<j}\left(\mu_{i}+\mu_{j}\right) H_{g}\left(\mu(\hat{i}, \hat{j}), \mu_{i}+\mu_{j}\right) \\
& +\frac{1}{2} \sum_{i=1}^{\ell} \sum_{\alpha+\beta=\mu_{i}} \alpha \beta\left(H_{g-1}(\mu(\hat{i}), \alpha, \beta)+\sum_{\substack{g_{1}+g_{2}=g \\
\nu_{1} \sqcup \nu_{2}=\mu(\hat{i})}} H_{g_{1}}\left(\nu_{1}, \alpha\right) H_{g_{2}}\left(\nu_{2}, \beta\right)\right) .
\end{aligned}
$$

Remark 3.3. Note that

$$
\ell(\mu(\hat{i}, \hat{j}))=\ell-2, \quad \ell\left(\nu_{1}\right)+\ell\left(\nu_{2}\right)=\ell(\mu(\hat{i}))=\ell-1 .
$$

Thus the complexity $2 g-2+\ell$ is one less for the coverings appearing on the RHS of 3.5 , which is the effect of $\partial / \partial s$ applied to $\mathbf{H}(s, \mathbf{p})$, except for the unstable geometry corresponding to $g_{i}=0$ and $\left|\nu_{i}\right|=0$ in the join terms. If we move the $(0,1)$-terms to the LHS, then the cut-and-join equation 3.5 becomes a topological recursion formula.

Let us first calculate the Laplace transform of the cut-and-join equation for the $\ell=1$ case to see what is involved. We then move on to the more general case, following 38 .

Proposition 3.4. The Laplace transform of the cut-and-join equation for the $\ell=1$ case gives the following equation:

$$
\begin{aligned}
& \sum_{n \leq 3 g-2}\left\langle\tau_{n} \Lambda_{g}^{\vee}(1)\right\rangle_{g, 1}\left[(2 g-1) \hat{\xi}_{n}(t)+\hat{\xi}_{n+1}(t)\left(1-\hat{\xi}_{-1}(t)\right)\right] \\
&=\frac{1}{2} \sum_{a+b \leq 3 g-4}\left[\left\langle\tau_{a} \tau_{b} \Lambda_{g-1}^{\vee}(1)\right\rangle_{g-1,2}\right. \\
&\left.+\sum_{g_{1}+g_{2}=g}^{\text {stable }}\left\langle\tau_{a} \Lambda_{g_{1}}^{\vee}(1)\right\rangle_{g_{1}, 1}\left\langle\tau_{b} \Lambda_{g_{2}}^{\vee}(1)\right\rangle_{g_{2}, 1}\right] \hat{\xi}_{a+1}(t) \hat{\xi}_{b+1}(t)
\end{aligned}
$$

Proof. The cut-and-join equation for $\ell=1$ is a simple equation

$$
(2 g-1+\mu) H_{g}(\mu)=\frac{1}{2} \sum_{\alpha+\beta=\mu} \alpha \beta\left(H_{g-1}(\alpha, \beta)+\sum_{g_{1}+g_{2}=g} H_{g_{1}}(\alpha) H_{g_{2}}(\beta)\right) .
$$

The Laplace transform of the LHS of 3.7 is

$$
\sum_{n \leq 3 g-2}\left\langle\tau_{n} \Lambda_{g}^{\vee}(1)\right\rangle_{g, 1}\left[(2 g-1) \hat{\xi}_{n}(t)+\hat{\xi}_{n+1}(t)\right] .
$$

When summing over $\mu$ to compute the Laplace transform of the RHS, we switch to sum over $\alpha$ and $\beta$ independently. The factor $1 / 2$ cancels the double count on 
the diagonal. Thus the Laplace transform of the stable geometries of the RHS is

$$
\begin{aligned}
\frac{1}{2} \sum_{a+b \leq 3 g-4}\left[\left\langle\tau_{a} \tau_{b} \Lambda_{g-1}^{\vee}(1)\right\rangle_{g-1,2}\right. & \\
& \left.+\sum_{g_{1}+g_{2}=g}^{\text {stable }}\left\langle\tau_{a} \Lambda_{g_{1}}^{\vee}(1)\right\rangle_{g_{1}, 1}\left\langle\tau_{b} \Lambda_{g_{2}}^{\vee}(1)\right\rangle_{g_{2}, 1}\right] \hat{\xi}_{a+1}(t) \hat{\xi}_{b+1}(t) .
\end{aligned}
$$

The unstable terms contained in the second summand of the RHS of (3.7) are the $g=0$ terms $H_{0}(\alpha) H_{g}(\beta)+H_{g}(\alpha) H_{0}(\beta)$. We calculate the Laplace transform of these unstable terms using (2.1). Since

$$
H_{0}(\alpha)=\frac{\alpha^{\alpha-2}}{\alpha !},
$$

the result is

$$
\sum_{a}\left\langle\tau_{a} \Lambda_{g}^{\vee}(1)\right\rangle_{g, 1} \hat{\xi}_{-1}(t) \hat{\xi}_{a+1}(t)
$$

This completes the proof.

Remark 3.5. We note that (3.6) is a polynomial equation of degree $2 n+2$. Since $\hat{\xi}_{-1}(t)=1-1 / t$, the leading term of $\hat{\xi}_{n+1}(t)$ is canceled in the formula.

To calculate the Laplace transform of the general case 3.5 , we need to deal with both of the unstable geometries $(g, \ell)=(0,1)$ and $(0,2)$. These are the exceptions for the general formula 2.14). Recall the $(0,1)$ case 2.1]. The formula

$$
\widehat{\mathcal{H}}_{0,1}(t)=\sum_{k=1}^{\infty} \frac{k^{k-2}}{k !} e^{-k(w+1)}=-\frac{1}{2 t^{2}}+c=\hat{\xi}_{-2}(t),
$$

where the constant $c$ is given by

$$
c=\sum_{k=1}^{\infty} \frac{k^{k-2}}{k !} e^{-k},
$$

is used in (3.6). The $(g, \ell)=(0,2)$ terms require a more careful computation. We shall see that these are the terms that exactly correspond to the terms involving the Cauchy differentiation kernel in the Bouchard-Mariño recursion.

Proposition 3.6. We have the following Laplace transformation formula:

$$
\begin{aligned}
\widehat{\mathcal{H}}_{0,2}\left(t_{1}, t_{2}\right) & =\sum_{\mu_{1}, \mu_{2} \geq 1} \frac{1}{\mu_{1}+\mu_{2}} \cdot \frac{\mu_{1}^{\mu_{1}}}{\mu_{1} !} \cdot \frac{\mu_{2}^{\mu_{2}}}{\mu_{2} !} e^{-\mu_{1}\left(w_{1}+1\right)} e^{-\mu_{2}\left(w_{2}+1\right)} \\
& =\log \left(\frac{\hat{\xi}_{-1}\left(t_{1}\right)-\hat{\xi}_{-1}\left(t_{2}\right)}{x_{1}-x_{2}}\right)-\hat{\xi}_{-1}\left(t_{1}\right)-\hat{\xi}_{-1}\left(t_{2}\right) .
\end{aligned}
$$


Proof. Since $x=e^{-(w+1)},(3.9)$ is equivalent to

$$
\sum_{\substack{\mu_{1}, \mu_{2} \geq 0 \\\left(\mu_{1}, \mu_{2}\right) \neq(0,0)}} \frac{1}{\mu_{1}+\mu_{2}} \cdot \frac{\mu_{1}^{\mu_{1}}}{\mu_{1} !} \cdot \frac{\mu_{2}^{\mu_{2}}}{\mu_{2} !} x_{1}^{\mu_{1}} x_{2}^{\mu_{2}}=\log \left(\sum_{k=1}^{\infty} \frac{k^{k-1}}{k !} \cdot \frac{x_{1}^{k}-x_{2}^{k}}{x_{1}-x_{2}}\right),
$$

where $\left|x_{1}\right|<e^{-1},\left|x_{2}\right|<e^{-1}$, and $0<\left|x_{1}-x_{2}\right|<e^{-1}$ so that the formula is an equality of holomorphic functions in $x_{1}$ and $x_{2}$. Define

$$
\phi\left(x_{1}, x_{2}\right) \stackrel{\text { def }}{=} \sum_{\substack{\mu_{1}, \mu_{2} \geq 0 \\\left(\mu_{1}, \mu_{2}\right) \neq(0,0)}} \frac{1}{\mu_{1}+\mu_{2}} \cdot \frac{\mu_{1}^{\mu_{1}}}{\mu_{1} !} \cdot \frac{\mu_{2}^{\mu_{2}}}{\mu_{2} !} x_{1}^{\mu_{1}} x_{2}^{\mu_{2}}-\log \left(\sum_{k=1}^{\infty} \frac{k^{k-1}}{k !} \cdot \frac{x_{1}^{k}-x_{2}^{k}}{x_{1}-x_{2}}\right) .
$$

Then

$$
\begin{aligned}
\phi(x, 0) & =\sum_{\mu_{1} \geq 1} \frac{\mu_{1}^{\mu_{1}-1}}{\mu_{1} !} x^{\mu_{1}}-\log \left(\sum_{k=1}^{\infty} \frac{k^{k-1}}{k !} \cdot x^{k-1}\right) \\
& =\hat{\xi}_{-1}(t)-\log \left(\frac{\hat{\xi}_{-1}(t)}{x}\right)=1-\frac{1}{t}-\log \left(1-\frac{1}{t}\right)+\log x \\
& =1-\frac{1}{t}-\log \left(1-\frac{1}{t}\right)-w-1=0
\end{aligned}
$$

due to 2.8). Here $t$ is restricted to the domain $\operatorname{Re}(t)>1$. Since

$$
\begin{aligned}
& x_{1} \frac{\partial}{\partial x_{1}} \log \left(\sum_{k=1}^{\infty} \frac{k^{k-1}}{k !} \cdot \frac{x_{1}^{k}-x_{2}^{k}}{x_{1}-x_{2}}\right) \\
& \quad=t_{1}^{2}\left(t_{1}-1\right) \frac{\partial}{\partial t_{1}} \log \left(\hat{\xi}_{-1}\left(t_{1}\right)-\hat{\xi}_{-1}\left(t_{2}\right)\right)-x_{1} \frac{\partial}{\partial x_{1}} \log \left(x_{1}-x_{2}\right) \\
& \quad=t_{1}^{2}\left(t_{1}-1\right) \frac{\partial}{\partial t_{1}} \log \left(-\frac{1}{t_{1}}+\frac{1}{t_{2}}\right)-\frac{x_{1}}{x_{1}-x_{2}}=\frac{t_{1} t_{2}\left(t_{1}-1\right)}{t_{1}-t_{2}}-\frac{x_{1}}{x_{1}-x_{2}}
\end{aligned}
$$

we have

$$
\begin{aligned}
& \left(x_{1} \frac{\partial}{\partial x_{1}}+x_{2} \frac{\partial}{\partial x_{2}}\right) \log \left(\sum_{k=1}^{\infty} \frac{k^{k-1}}{k !} \cdot \frac{x_{1}^{k}-x_{2}^{k}}{x_{1}-x_{2}}\right) \\
& =\frac{t_{1} t_{2}\left(t_{1}-1\right)-t_{1} t_{2}\left(t_{2}-1\right)}{t_{1}-t_{2}}-\frac{x_{1}-x_{2}}{x_{1}-x_{2}}=t_{1} t_{2}-1=\hat{\xi}_{0}\left(t_{1}\right) \hat{\xi}_{0}\left(t_{2}\right)+\hat{\xi}_{0}\left(t_{1}\right)+\hat{\xi}_{0}\left(t_{2}\right) .
\end{aligned}
$$

On the other hand, we also have

$$
\begin{aligned}
\left(x_{1} \frac{\partial}{\partial x_{1}}+x_{2} \frac{\partial}{\partial x_{2}}\right) \sum_{\substack{\mu_{1}, \mu_{2} \geq 0 \\
\left(\mu_{1}, \mu_{2}\right) \neq(0,0)}} \frac{1}{\mu_{1}+\mu_{2}} & \cdot \frac{\mu_{1}^{\mu_{1}}}{\mu_{1} !} \cdot \frac{\mu_{2}^{\mu_{2}}}{\mu_{2} !} x_{1}^{\mu_{1}} x_{2}^{\mu_{2}} \\
& =\hat{\xi}_{0}\left(t_{1}\right) \hat{\xi}_{0}\left(t_{2}\right)+\hat{\xi}_{0}\left(t_{1}\right)+\hat{\xi}_{0}\left(t_{2}\right) .
\end{aligned}
$$


Therefore,

$$
\left(x_{1} \frac{\partial}{\partial x_{1}}+x_{2} \frac{\partial}{\partial x_{2}}\right) \phi\left(x_{1}, x_{2}\right)=0 .
$$

Note that $\phi\left(x_{1}, x_{2}\right)$ is a holomorphic function in $x_{1}$ and $x_{2}$. Therefore, it has a series expansion in homogeneous polynomials around $(0,0)$. Since a homogeneous polynomial in $x_{1}$ and $x_{2}$ of degree $n$ is an eigenvector of the differential operator $x_{1} \frac{\partial}{\partial x_{1}}+x_{2} \frac{\partial}{\partial x_{2}}$ belonging to the eigenvalue $n$, the only holomorphic solution to the Euler differential equation (3.11) is a constant. But since $\phi\left(x_{1}, 0\right)=0$, we conclude that $\phi\left(x_{1}, x_{2}\right)=0$. This completes the proof of 3.10$)$, and hence of the proposition.

The following polynomial recursion formula was established in 38 . Since each of the polynomials $\widehat{\mathcal{H}}_{g, \ell}\left(t_{L}\right)$ in 3.12 satisfies the stability condition $2 g-2+\ell>0$, it is equivalent to 1.2 after expanding the generating functions using (2.14).

Theorem 3.7 ([38]). The Laplace transform of the cut-and-join equation (3.5) produces the following polynomial equation on the Lambert curve:

$$
\begin{gathered}
\left(2 g-2+\ell+\sum_{i=1}^{\ell}\left(1-\hat{\xi}_{-1}\left(t_{i}\right)\right) t_{i}^{2}\left(t_{i}-1\right) \frac{\partial}{\partial t_{i}}\right) \widehat{\mathcal{H}}_{g, \ell}\left(t_{1}, \ldots, t_{\ell}\right) \\
=\sum_{i<j} t_{i} t_{j} \frac{t_{i}^{2}\left(t_{i}-1\right)^{2} \frac{\partial}{\partial t_{i}} \widehat{\mathcal{H}}_{g, \ell-1}\left(\widehat{t_{j}}\right)-t_{j}^{2}\left(t_{j}-1\right)^{2} \frac{\partial}{\partial t_{j}} \widehat{\mathcal{H}}_{g, \ell-1}\left(\widehat{t_{i}}\right)}{t_{i}-t_{j}} \\
\quad-\sum_{i \neq j} t_{i}^{3}\left(t_{i}-1\right) \frac{\partial}{\partial t_{i}} \widehat{\mathcal{H}}_{g, \ell-1}\left(\widehat{t_{j}}\right) \\
+\frac{1}{2} \sum_{i=1}^{\ell}\left[u_{1}^{2}\left(u_{1}-1\right) u_{2}^{2}\left(u_{2}-1\right) \frac{\partial^{2}}{\partial u_{1} \partial u_{2}} \widehat{\mathcal{H}}_{g-1, \ell+1}\left(u_{1}, u_{2}, t_{L \backslash\{i\}}\right)\right]_{u_{1}=u_{2}=t_{i}} \\
+\frac{1}{2} \sum_{i=1}^{\ell} \sum_{\substack{g_{1}+g_{2}=g \\
\text { stable }}} t_{i}^{2}\left(t_{i}-1\right) \frac{\partial}{\partial t_{i}} \widehat{\mathcal{H}}_{g_{1},|J|+1}\left(t_{i}, t_{J}\right) \cdot t_{i}^{2}\left(t_{i}-1\right) \frac{\partial}{\partial t_{i}} \widehat{\mathcal{H}}_{g_{2},|K|+1}\left(t_{i}, t_{K}\right),
\end{gathered}
$$

where

$$
\widehat{\mathcal{H}}_{g, \ell-1}\left(\widehat{t}_{j}\right)=\widehat{\mathcal{H}}_{g, \ell-1}\left(t_{1}, \ldots, \widehat{t}_{j}, \ldots, t_{\ell}\right)
$$

In the last sum each term is restricted to satisfy the stability conditions $2 g_{1}-1+$ $|J|>0$ and $2 g_{2}-1+|K|>0$.

Remark 3.8. The polynomial equation 3.12 is equivalent to the original cutand-join equation 3.5 . Note that the topological recursion structure of 3.12 
is exactly the same as (1.5). Although 3.12 contains more terms, all functions involved are polynomials that are easy to calculate from 2.11, whereas 1.5 requires computation of the involution $s(t)$ of $(2.9)$ and infinite series expansions.

Remark 3.9. It is an easy task to deduce the Witten-Kontsevich theorem, i.e., the Virasoro constraint condition for the $\psi$-class intersection numbers [44, 27, from (3.12). Let us use the normalized notation $\sigma_{n}=(2 n+1) ! ! \tau_{n}$ for the $\psi$-class intersections. Then the formula according to Dijkgraaf, Verlinde and Verlinde [7] is

$$
\begin{aligned}
\left\langle\sigma_{n} \sigma_{n_{L}}\right\rangle_{g, \ell+1} & \\
= & \frac{1}{2} \sum_{a+b=n-2}\left\langle\sigma_{a} \sigma_{b} \sigma_{n_{L}}\right\rangle_{g-1, \ell+2}+\sum_{i \in L}\left(2 n_{i}+1\right)\left\langle\sigma_{n+n_{i}-1} \sigma_{n_{L \backslash\{i\}}}\right\rangle_{g, \ell} \\
& +\frac{1}{2} \sum_{\substack{g_{1}+g_{2}=g \\
I \sqcup J=L}}^{\text {stable }} \sum_{a+b=n-2}\left\langle\sigma_{a} \sigma_{n_{I}}\right\rangle_{g_{1},|I|+1} \cdot\left\langle\sigma_{b} \sigma_{n_{J}}\right\rangle_{g_{2},|J|+1} .
\end{aligned}
$$

This is exactly the relation of the homogeneous top degree terms of (3.12), after canceling the highest degree terms coming from $\hat{\xi}_{n_{i}+1}\left(t_{i}\right)$ in the LHS 38. This derivation is in the same spirit as those found in 4, 26, 41, though the argument is much clearer due to the polynomial nature of our equation.

\section{§4. The Bouchard-Mariño recursion formula for Hurwitz numbers}

In this section we present the precise statement of the Bouchard-Mariño conjecture on Hurwitz numbers.

Recall the function we introduced in $(2.6)$ :

$$
t=t(x)=1+\sum_{k=1}^{\infty} \frac{k^{k}}{k !} x^{k} .
$$

This is closely related to the Lambert $W$-function

$$
W(x)=-\sum_{k=1}^{\infty} \frac{k^{k-1}}{k !}(-x)^{k} .
$$

By abuse of terminology, we also call the function $t(x)$ of 4.1 the Lambert function. The power series 4.1 has the radius of convergence $1 / e$, and its inverse function is given by

$$
x=x(t)=\frac{1}{e}\left(1-\frac{1}{t}\right) e^{1 / t} .
$$

Motivated by the Lambert $W$-function, a plane analytic curve

$$
C=\{(x, t) \mid x=x(t)\} \subset \mathbb{C}^{*} \times \mathbb{C}^{*}
$$


is introduced in [3, which is exactly the Lambert curve (1.3). We denote by $\pi$ : $C \rightarrow \mathbb{C}$ the $x$-projection. Bouchard-Mariño $[3$ then defines a tower of polynomial differentials on the Lambert curve $C$ by

$$
\xi_{n}(t)=\frac{d}{d t}\left[t^{2}(t-1) \xi_{n-1}(t)\right]
$$

with the initial condition

$$
\xi_{0}(t)=d t
$$

It is obvious from 4.5 and 4.6 that for $n \geq 0, \xi_{n}(t)$ is a polynomial 1-form of degree $2 n$ with a general expression

$\xi_{n}(t)=t^{n}\left[(2 n+1) ! ! t^{n}-\left(\frac{(2 n+3) ! !}{3}-(2 n+1) ! !\right) t^{n-1}+\cdots+(-1)^{n}(n+1) !\right] d t$.

All the coefficients of $\xi_{n}(t)$ have a combinatorial meaning called the second order reciprocal Stirling numbers. As we will note below, the leading coefficient is responsible for the Witten-Kontsevich theorem on the cotangent class intersections, and the lowest coefficient is related to the $\lambda_{g}$-formula [38. For convenience, we also use $\xi_{-1}(t)=t^{-2} d t$ and $\xi_{-2}(t)=t^{-3} d t$.

Remark 4.1. The polynomial $\hat{\xi}_{n}(t)$ of 2.10 is a primitive of $\xi_{n}(t)$ :

$$
d \hat{\xi}_{n}(t)=\xi_{n}(t) .
$$

Definition 4.2. Let us call the symmetric polynomial differential form

$$
d^{\otimes \ell} \widehat{\mathcal{H}}_{g, \ell}\left(t_{1}, \ldots, t_{\ell}\right)=\sum_{n_{1}+\cdots+n_{\ell} \leq 3 g-3+\ell}\left\langle\tau_{n_{1}} \cdots \tau_{n_{\ell}} \Lambda_{g}^{\vee}(1)\right\rangle \bigotimes_{i=1}^{\ell} \xi_{n_{i}}\left(t_{i}\right)
$$

on $C^{\ell}$ the Hurwitz differential of type $(g, \ell)$.

Remark 4.3. Our $\xi_{n}(t)$ is exactly the same as the $\zeta_{n}(y)$-differential of [3. However, this mere coordinate change happens to be essential. Indeed, the fact that our expression is a polynomial in t-variables allows us to calculate the residues in the Bouchard-Mariño formula in Section 5 .

Remark 4.4. The degree of $d^{\otimes \ell} \widehat{\mathcal{H}}_{g, \ell}\left(t_{1}, \ldots, t_{\ell}\right)$ is $2(3 g-3+\ell)$, and the homogeneous top degree terms give a generating function of the $\psi$-class intersection numbers

$$
\sum_{n_{1}+\cdots+n_{\ell}=3 g-3+\ell}\left\langle\tau_{n_{1}} \cdots \tau_{n_{\ell}}\right\rangle \prod_{i=1}^{\ell}\left(2 n_{i}+1\right) ! ! t_{i}^{2 n_{i}} \bigotimes_{i=1}^{\ell} d t_{i}
$$


The homogeneous lowest degree terms of $d^{\otimes \ell} \widehat{\mathcal{H}}_{g, \ell}\left(t_{1}, \ldots, t_{\ell}\right)$ are

$$
(-1)^{3 g-3+\ell} \sum_{n_{1}+\cdots+n_{\ell}=2 g-3+\ell}\left\langle\tau_{n_{1}} \cdots \tau_{n_{\ell}} \lambda_{g}\right\rangle \prod_{i=1}^{\ell}\left(n_{i}+1\right) ! t_{i}^{n_{i}} \bigotimes_{i=1}^{\ell} d t_{i} .
$$

The combinatorial coefficients of the $\lambda_{g}$-formula [15, 16] can be directly deduced from the topological recursion formula 1.2 38, explaining the mechanism found in [20].

Remark 4.5. The unstable Hurwitz differentials follow from 2.1$)$ and $(3.9)$. They are

$$
\begin{aligned}
& d \widehat{\mathcal{H}}_{0,1}(t)=\frac{1}{t^{3}} d t=\xi_{-2}(t) ; \\
& d^{\otimes 2} \widehat{\mathcal{H}}_{0,2}\left(t_{1}, t_{2}\right)=\frac{d t_{1} \otimes d t_{2}}{\left(t_{1}-t_{2}\right)^{2}}-\pi^{*} \frac{d x_{1} \otimes d x_{2}}{\left(x_{1}-x_{2}\right)^{2}} .
\end{aligned}
$$

Remark 4.6. The simplest stable Hurwitz differentials are given by

$$
\begin{aligned}
& d^{\otimes 3} \widehat{\mathcal{H}}_{0,3}\left(t_{1}, t_{2}, t_{3}\right)=d t_{1} \otimes d t_{2} \otimes d t_{3} ; \\
& d \widehat{\mathcal{H}}_{1,1}(t)=\frac{1}{24}\left(-\xi_{0}(t)+\xi_{1}(t)\right)=\frac{1}{24}(t-1)(3 t+1) d t .
\end{aligned}
$$

The amazing insight of Bouchard and Mariño [3] is that the Hurwitz differentials of Definition 4.2 should satisfy the topological recursion relation of Eynard and Orantin 13 based on the analytic curve $C$ of $(4.4$ as the spectral curve. Since the topological recursion utilizes the critical behavior of the $x$-projection $\pi: C \rightarrow \mathbb{C}^{*}$, let us examine the local structure of $C$ around its critical points. Let $z=-1 / t$ be a coordinate of $C$ centered at $t=\infty$. The Lambert curve is then given by

$$
x=\frac{1}{e}(1+z) e^{-z}
$$

We see that the $x$-projection $\pi: C \rightarrow \mathbb{C}^{*}$ has a unique critical point $q_{0}$ at $z=0$. Locally around $q_{0}$ the curve $C$ is a double cover of $\mathbb{C}$ branched at $q_{0}$. For a point $q \in C$ near $q_{0}$, let us denote by $\bar{q}$ the Galois conjugate point on $C$ that has the same $x$-coordinate. Let $S(z)$ be the local deck transformation of the covering $\pi: C \rightarrow \mathbb{C}^{*}$. Its defining equation

$$
S(z)-\log (1+S(z))=z-\log (1+z)=\sum_{m=2}^{\infty} \frac{(-1)^{m}}{m} z^{m}
$$

has a unique analytic solution other than $z$ itself, which has a branch cut along $(-\infty,-1]$. We note that $S$ is an involution, $S(S(z))=z$, and has a Taylor expansion $S(z)=-z+\frac{2}{3} z^{2}-\frac{4}{9} z^{3}+\frac{44}{135} z^{4}-\frac{104}{405} z^{5}+\frac{40}{189} z^{6}-\frac{7648}{42525} z^{7}+\frac{2848}{18225} z^{8}+O\left(z^{9}\right)$ 
for $|z|<1$. In terms of the $t$-coordinate, the involution corresponds to $s(t)$ of $2.9 p$ :

$$
\left\{\begin{array}{l}
t(q)=-1 / z=t, \\
t(\bar{q})=-1 / S(z)=s(t) .
\end{array}\right.
$$

The equation 4.11) defining $S(z)$ translates into a relation

$$
\frac{d t}{t^{2}(t-1)}=\frac{d s(t)}{s(t)^{2}(s(t)-1)}=-d w=-v d v=\pi^{*}\left(\frac{d x}{x}\right) .
$$

Using the global coordinate $t$ of the Lambert curve $C$, the Cauchy differentiation kernel (called the Bergman kernel in [13, 3]) is defined by

$$
B\left(t_{1}, t_{2}\right)=\frac{d t_{1} \otimes d t_{2}}{\left(t_{1}-t_{2}\right)^{2}}=d_{t_{1}} d_{t_{2}} \log \left(t_{1}-t_{2}\right) .
$$

We have already encountered it in $(4.9)$ in the expression of $\mathcal{H}_{0,2}\left(t_{1}, t_{2}\right)$. Following [13, define a 1-form on $C$ by

$$
\begin{aligned}
d E\left(q, \bar{q}, t_{2}\right) & =\frac{1}{2} \int_{q}^{\bar{q}} B\left(\cdot, t_{2}\right)=\frac{1}{2}\left(\frac{1}{t_{1}-t_{2}}-\frac{1}{s\left(t_{1}\right)-t_{2}}\right) d t_{2} \\
& =\frac{1}{2}\left(\hat{\xi}_{-1}\left(s\left(t_{1}\right)-t_{2}\right)-\hat{\xi}_{-1}\left(t_{1}-t_{2}\right)\right) d t_{2},
\end{aligned}
$$

where the integral is taken with respect to the first variable of $B\left(t_{1}, t_{2}\right)$ along any path from $q$ to $\bar{q}$. The natural holomorphic symplectic form on $\mathbb{C}^{*} \times \mathbb{C}^{*}$ is given by

$$
\Omega=d \log y \wedge d \log x=d \log \left(1-\frac{1}{t}\right) \wedge d \log x .
$$

Again following [3, 13], let us introduce another 1-form on the curve $C$ by

$$
\omega(q, \bar{q})=\int_{q}^{\bar{q}} \Omega(\cdot, x(q))=\left(\frac{1}{t}-\frac{1}{s(t)}\right) \frac{d t}{t^{2}(t-1)}=\left(\hat{\xi}_{-1}(s(t))-\hat{\xi}_{-1}(t)\right) \frac{d t}{t^{2}(t-1)} .
$$

The kernel operator is defined as the quotient

$$
K\left(t_{1}, t_{2}\right)=\frac{d E\left(q, \bar{q}, t_{2}\right)}{\omega(q, \bar{q})}=\frac{1}{2} \cdot \frac{t_{1}}{t_{1}-t_{2}} \cdot \frac{s\left(t_{1}\right)}{s\left(t_{1}\right)-t_{2}} \cdot \frac{t_{1}^{2}\left(t_{1}-1\right)}{d t_{1}} \otimes d t_{2},
$$

which is a linear algebraic operator acting on symmetric differential forms on $C^{\ell}$ by replacing $d t_{1}$ with $d t_{2}$. We note that

$$
K\left(t_{1}, t_{2}\right)=K\left(s\left(t_{1}\right), t_{2}\right),
$$

which follows from 4.12. In the $z$-coordinate, the kernel has the expression 


$$
\begin{aligned}
K= & -\frac{1}{2} \cdot \frac{1+z}{z} \cdot \frac{1}{(1+z t)(1+S(z) t)} \cdot d t \otimes \frac{1}{d z} \\
= & -\frac{1}{2} \cdot \frac{1+z}{z}\left(\sum_{m=0}^{\infty}(-1)^{m} \cdot \frac{z^{m+1}-S(z)^{m+1}}{z-S(z)} \cdot t^{m}\right) d t \otimes \frac{1}{d z} \\
= & -\frac{1}{2}\left(\frac{1}{z}+1+\frac{1}{3}(3 t-2) t z+\frac{1}{9}(3 t-2) t z^{2}\right. \\
& \left.+\frac{1}{135}\left(135 t^{3}-180 t^{2}+30 t+16\right) t z^{3}+\cdots\right) d t \otimes \frac{1}{d z} .
\end{aligned}
$$

Definition 4.7. The topological recursion formula is an inductive mechanism of defining a symmetric $\ell$-form

$$
W_{g, \ell}\left(t_{L}\right)=W_{g, \ell}\left(t_{1}, \ldots, t_{\ell}\right)
$$

on $C^{\ell}$ for any given $g$ and $\ell$ subject to $2 g-2+\ell>0$ by

$$
\begin{aligned}
& W_{g, \ell+1}\left(t_{0}, t_{L}\right)=-\frac{1}{2 \pi i} \oint_{\gamma_{\infty}}\left[K ( t , t _ { 0 } ) \left(W_{g-1, \ell+2}\left(t, s(t), t_{L}\right)\right.\right. \\
& +\sum_{i=1}^{\ell}\left(W_{g, \ell}\left(t, t_{L \backslash\{i\}}\right) \otimes B\left(s(t), t_{i}\right)+W_{g, \ell}\left(s(t), t_{L \backslash\{i\}}\right) \otimes B\left(t, t_{i}\right)\right) \\
& \left.\left.\quad+\sum_{g_{1}+g_{2}=g, I \sqcup J=L}^{\text {stable terms }} W_{g_{1},|I|+1}\left(t, t_{I}\right) \otimes W_{g_{2},|J|+1}\left(s(t), t_{J}\right)\right)\right] .
\end{aligned}
$$

Here $t_{I}=\left(t_{i}\right)_{i \in I}$ for a subset $I \subset L=\{1, \ldots, \ell\}$, and the last sum is taken over all partitions of $g$ and disjoint decompositions $I \sqcup J=L$ subject to the stability condition $2 g_{1}-1+|I|>0$ and $2 g_{2}-1+|J|>0$. The integration is taken with respect to $d t$ on the contour $\gamma_{\infty}$, which is a positively oriented loop of large radius in the complex $t$-plane so that $|t|>\max \left(\left|t_{0}\right|,\left|s\left(t_{0}\right)\right|\right)$ for $t \in \gamma_{\infty}$.

Now we can state the Bouchard-Mariño conjecture, which we prove in Section 7

Conjecture 4.8 (Bouchard-Mariño Conjecture [3]). For every $g$ and $\ell$ subject to the stability condition $2 g-2+\ell>0$, the topological recursion formula 4.16) with the initial condition

$$
\left\{\begin{array}{l}
W_{0,3}\left(t_{1}, t_{2}, t_{3}\right)=d t_{1} \otimes d t_{2} \otimes d t_{3}, \\
W_{1,1}\left(t_{1}\right)=\frac{1}{24}\left(t_{1}-1\right)\left(3 t_{1}+1\right) d t_{1},
\end{array}\right.
$$

gives the Hurwitz differential

$$
W_{g, \ell}\left(t_{1}, \ldots, t_{\ell}\right)=d^{\otimes \ell} \widehat{\mathcal{H}}_{g, \ell}\left(t_{1}, \ldots, t_{\ell}\right) .
$$


Remark 4.9. In the literature [3, 13, the topological recursion is written as

$$
\begin{aligned}
W_{g, \ell+1}\left(t_{0}, t_{L}\right) & =\operatorname{Res}_{q=\bar{q}}\left[\frac { d E ( q , \overline { q } , t _ { 0 } ) } { \omega ( q , \overline { q } ) } \left(W_{g-1, \ell+2}\left(t(q), t(\bar{q}), t_{L}\right)\right.\right. \\
+ & \left.\left.\sum_{g_{1}+g_{2}=g, I \sqcup J=L} W_{g_{1},|I|+1}\left(t(q), t_{I}\right) \otimes W_{g_{2},|J|+1}\left(t(\bar{q}), t_{J}\right)\right)\right],
\end{aligned}
$$

including all possible terms in the second line, with the initial condition

$$
W_{0,1}\left(t_{1}\right)=0, \quad W_{0,2}\left(t_{1}, t_{2}\right)=B\left(t_{1}, t_{2}\right) .
$$

If we single out the stable terms from (4.18), then we obtain 4.16). Although the initial values of $W_{g, \ell}$ given in 4.19$)$ are different from $(4.8)$ and $(4.9)$, the advantage of (4.18) is that we can include (4.17) as a consequence of the recursion.

Remark 4.10. It is established in [13] that a solution of the topological recursion is a symmetric differential form in general. In our case, the RHS of the recursion formula 4.16 does not appear to be symmetric in $t_{0}, t_{1}, \ldots, t_{\ell}$. We note that our proof of the formula establishes this symmetry because the Hurwitz differential is a symmetric polynomial. This situation is again strikingly similar to the Mizrakhani recursion [35, 36, where the symmetry appears not as a consequence of the recursion, but rather as the geometric nature of the quantity the recursion calculates, namely, the Weil-Petersson volume of the moduli space of bordered hyperbolic surfaces.

\section{$\S 5$. Residue calculation}

In this section we calculate the residues appearing in the recursion formula 4.16). It turns out to be equivalent to the direct image operation with respect to the projection $\pi: C \rightarrow \mathbb{C}$.

Recall that the kernel $K\left(t, t_{0}\right)$ is a rational expression in terms of $t, s(t)$ and $t_{0}$. The function $s(t)$ is an involution, $s(s(t))=t$, defined outside of the slit [0,1] of the complex $t$-plane, with logarithmic singularities at 0 and 1 . Our idea of computing the residue is to decompose the integration over the loop $\gamma_{\infty}$ into the sum of integrations over $\gamma_{\infty}-\gamma_{[0,1]}$ and $\gamma_{[0,1]}$, where $\gamma_{[0,1]}$ is a positively oriented thin loop containing the interval $[0,1]$.

Definition 5.1. For a Laurent series $\sum_{n \in \mathbb{Z}} a_{n} t^{n}$, we denote

$$
\left[\sum_{n \in \mathbb{Z}} a_{n} t^{n}\right]_{+}=\sum_{n \geq 0} a_{n} t^{n} .
$$




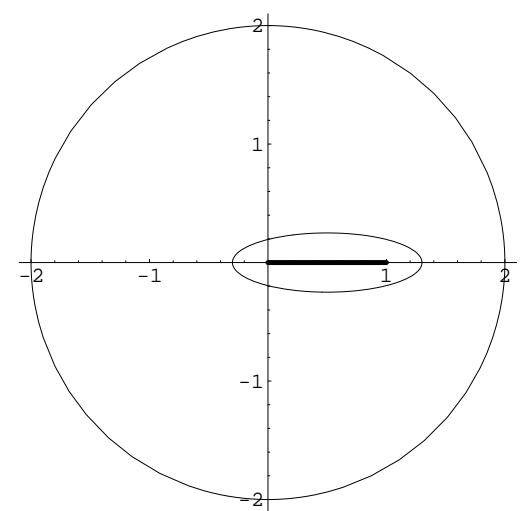

Figure 5.1. The contours of integration. $\gamma_{\infty}$ is the circle of a large radius, and $\gamma_{[0,1]}$ is the thin loop surrounding the closed interval $[0,1]$.

Theorem 5.2. In terms of the primitives $\hat{\xi}_{n}(t)$, we have

$$
\begin{aligned}
R_{a, b}(t) & =-\frac{1}{2 \pi i} \oint_{\gamma_{\infty}} K\left(t^{\prime}, t\right) \xi_{a}\left(t^{\prime}\right) \xi_{b}\left(s\left(t^{\prime}\right)\right) \\
& =\frac{1}{2}\left[\frac{t s(t)}{t-s(t)}\left(\xi_{a}(t) \hat{\xi}_{b+1}(s(t))+\hat{\xi}_{a+1}(s(t)) \xi_{b}(t)\right)\right]_{+} .
\end{aligned}
$$

Similarly,

$$
\begin{aligned}
R_{n}\left(t, t_{i}\right) & =-\frac{1}{2 \pi i} \oint_{\gamma_{\infty}} K\left(t^{\prime}, t\right)\left(\xi_{n}\left(t^{\prime}\right) B\left(s\left(t^{\prime}\right), t_{i}\right)+\xi_{n}\left(s\left(t^{\prime}\right)\right) B\left(t^{\prime}, t_{i}\right)\right) \\
& =\left[\frac{t s(t)}{t-s(t)}\left(\hat{\xi}_{n+1}(t) B\left(s(t), t_{i}\right)+\hat{\xi}_{n+1}(s(t)) B\left(t, t_{i}\right)\right)\right]_{+} .
\end{aligned}
$$

Proof. In terms of the original $z$-coordinate of [3], the residue $R_{a, b}(t)$ is simply the coefficient of $z^{-1}$ in $K\left(t^{\prime}, t\right) \xi_{a}\left(t^{\prime}\right) \xi_{b}\left(s\left(t^{\prime}\right)\right)$, after expanding it in the Laurent series in $z$. Since $\xi_{n}\left(t^{\prime}\right)$ is a polynomial in $t^{\prime}=-1 / z$, the contribution to the $z^{-1}$ term in the expression is a polynomial in $t$ because of the $z$-expansion formula 4.15 for the kernel $K$. Thus we know that $R_{a, b}(t)$ is a polynomial in $t$.

Let us write $\xi_{n}(t)=f_{n}(t) d t$, and let $\gamma_{[0,1]}$ be a positively oriented loop containing the slit $[0,1]$, as in Figure 5.1 . On this compact set we have a bound

$$
\left|\frac{t s(t)}{t-s(t)} t^{2}(t-1) s^{\prime}(t) f_{a}(t) f_{b}(s(t))\right|<M,
$$

since the function is holomorphic outside $[0,1]$. Choose $|t| \gg 1$. Then we have 


$$
\begin{aligned}
\mid-\frac{1}{2 \pi i} \oint_{\gamma_{[0,1]}} & K\left(t^{\prime}, t\right) \xi_{a}\left(t^{\prime}\right) \xi_{b}\left(s\left(t^{\prime}\right)\right) \mid \\
= & \mid-\frac{1}{2 \pi i} \oint_{\gamma_{[0,1]}} \frac{1}{2}\left(\frac{1}{t^{\prime}-t}-\frac{1}{s\left(t^{\prime}\right)-t}\right) \frac{t^{\prime} s\left(t^{\prime}\right)}{s\left(t^{\prime}\right)-t^{\prime}} \\
& \times t^{\prime 2}\left(t^{\prime}-1\right) s^{\prime}\left(t^{\prime}\right) f_{a}\left(t^{\prime}\right) f_{b}\left(s\left(t^{\prime}\right)\right) d t^{\prime} \mid \otimes d t \\
& <\frac{M}{2 \pi} \oint_{\gamma_{[0,1]}} \frac{1}{2}\left|\frac{1}{t^{\prime}-t}-\frac{1}{s\left(t^{\prime}\right)-t}\right| d t^{\prime} \otimes d t \sim \frac{M}{4 \pi|t|} d t .
\end{aligned}
$$

Therefore,

$$
\begin{aligned}
-\frac{1}{2 \pi i} \oint_{\gamma_{\infty}} K\left(t^{\prime}, t\right) \xi_{a}\left(t^{\prime}\right) & \xi_{b}\left(s\left(t^{\prime}\right)\right) \\
& =-\frac{1}{2 \pi i} \oint_{\gamma_{\infty}-\gamma_{[0,1]}} K\left(t^{\prime}, t\right) \xi_{a}\left(t^{\prime}\right) \xi_{b}\left(s\left(t^{\prime}\right)\right)+O\left(t^{-1}\right) \\
& =\left[-\frac{1}{2 \pi i} \oint_{\gamma_{\infty}-\gamma_{[0,1]}} K\left(t^{\prime}, t\right) \xi_{a}\left(t^{\prime}\right) \xi_{b}\left(s\left(t^{\prime}\right)\right)\right]_{+} .
\end{aligned}
$$

Noticing the relation 4.12 and the fact that $s(t)$ is an involution, we obtain

$$
\begin{aligned}
&-\frac{1}{2 \pi i} \oint_{\gamma_{\infty}-\gamma_{[0,1]}} K\left(t^{\prime}, t\right) \xi_{a}\left(t^{\prime}\right) \xi_{b}\left(s\left(t^{\prime}\right)\right) \\
&=-\frac{1}{2 \pi i} \oint_{\gamma_{\infty}-\gamma_{[0,1]}} \frac{1}{2}\left(\frac{1}{t^{\prime}-t}-\frac{1}{s\left(t^{\prime}\right)-t}\right) \frac{t^{\prime} s\left(t^{\prime}\right)}{s\left(t^{\prime}\right)-t^{\prime}} \\
& \times t^{\prime 2}\left(t^{\prime}-1\right) s^{\prime}\left(t^{\prime}\right) f_{a}\left(t^{\prime}\right) f_{b}\left(s\left(t^{\prime}\right)\right) d t^{\prime} \otimes d t \\
&=-\frac{1}{2 \pi i} \oint_{\gamma_{\infty}-\gamma_{[0,1]}} \frac{1}{2} \cdot \frac{1}{t^{\prime}-t} \cdot \frac{t^{\prime} s\left(t^{\prime}\right)}{s\left(t^{\prime}\right)-t^{\prime}} \cdot t^{\prime 2}\left(t^{\prime}-1\right) s^{\prime}\left(t^{\prime}\right) f_{a}\left(t^{\prime}\right) f_{b}\left(s\left(t^{\prime}\right)\right) d t^{\prime} \otimes d t \\
&+\frac{1}{2 \pi i} \oint_{s\left(\gamma_{\infty}\right)-s\left(\gamma_{[0,1]}\right)} \frac{1}{2} \cdot \frac{1}{s\left(t^{\prime}\right)-t} \cdot \frac{t^{\prime} s\left(t^{\prime}\right)}{s\left(t^{\prime}\right)-t^{\prime}} \\
& \times t^{\prime 2}\left(t^{\prime}-1\right) f_{a}\left(t^{\prime}\right) f_{b}\left(s\left(t^{\prime}\right)\right) d s\left(t^{\prime}\right) \otimes d t \\
&= \frac{1}{2} \cdot \frac{t s(t)}{t-s(t)} \cdot t^{2}(t-1) s^{\prime}(t) f_{a}(t) f_{b}(s(t)) d t \\
&+\frac{1}{2} \cdot \frac{s(t) t}{t-s(t)} \cdot s(t)^{2}(s(t)-1) f_{a}(s(t)) f_{b}(t) d t \\
&= \frac{t s(t)}{t-s(t)} t^{2}(t-1) s^{\prime}(t) \frac{f_{a}(t) f_{b}(s(t))+f_{a}(s(t)) f_{b}(t)}{2} d t \\
&= \frac{1}{2} \cdot \frac{t s(t)}{t-s(t)}\left(\xi_{a}(t) \hat{\xi}_{b+1}(s(t))+\hat{\xi}_{a+1}(s(t)) \xi_{b}(t)\right)
\end{aligned}
$$




$$
\begin{aligned}
& =\frac{1}{2} \cdot \frac{t s(t)}{t-s(t)}\left(\hat{\xi}_{a}^{\prime}(t) \hat{\xi}_{b+1}(s(t))+\hat{\xi}_{a+1}(s(t)) \hat{\xi}_{b}^{\prime}(t)\right) d t \\
& =\frac{1}{2} \cdot \frac{t s(t)}{t-s(t)}\left(\hat{\xi}_{a+1}(t) \hat{\xi}_{b+1}(s(t))+\hat{\xi}_{a+1}(s(t)) \hat{\xi}_{b+1}(t)\right) \frac{d t}{t^{2}(t-1)} .
\end{aligned}
$$

Here we used 2.11 and 4.12 at the last step. The proof of the second residue formula is exactly the same.

Remark 5.3. The equation for the kernel 4.14 implies

$$
R_{a, b}(t)=R_{b, a}(t)=-\left[R_{a, b}(s(t))\right]_{+} .
$$

Let us define polynomials $P_{a, b}(t)$ and $P_{n}\left(t, t_{i}\right)$ by

$$
\begin{gathered}
P_{a, b}(t) d t=\frac{1}{2}\left[\frac{t s(t)}{t-s(t)} \frac{d t}{t^{2}(t-1)}\left(\hat{\xi}_{a+1}(t) \hat{\xi}_{b+1}(s(t))+\hat{\xi}_{a+1}(s(t)) \hat{\xi}_{b+1}(t)\right)\right]_{+} \\
P_{n}\left(t, t_{i}\right) d t \otimes d t_{i}=d_{t_{i}}\left[\frac{t s(t)}{t-s(t)}\left(\frac{\hat{\xi}_{n+1}(t) d s(t)}{s(t)-t_{i}}+\frac{\hat{\xi}_{n+1}(s(t)) d t}{t-t_{i}}\right)\right]_{+}
\end{gathered}
$$

Obviously $\operatorname{deg} P_{a, b}(t)=2(a+b+2)$. To calculate $P_{n}\left(t, t_{i}\right)$, we use the Laurent series expansion

$$
\frac{1}{t-t_{i}}=\frac{1}{t} \sum_{k=0}^{\infty}\left(\frac{t_{i}}{t}\right)^{k},
$$

and take the polynomial part in $t$. We note that it is automatically a polynomial in $t_{i}$ as well. We thus see that $\operatorname{deg} P_{n}\left(t, t_{i}\right)=2 n+2$ in each variable.

Theorem 5.4. The topological recursion formula 4.16 is equivalent to the following equality of symmetric differential forms in $\ell+1$ variables with polynomial coefficients:

$$
\begin{aligned}
& \sum_{n, n_{L}}\left\langle\tau_{n} \tau_{n_{L}} \Lambda_{g}^{\vee}(1)\right\rangle_{g, \ell+1} d \hat{\xi}_{n}(t) \otimes d \hat{\xi}_{n_{L}}\left(t_{L}\right) \\
& =\sum_{i=1}^{\ell} \sum_{m, n_{L \backslash\{i\}}}\left\langle\tau_{m} \tau_{n_{L \backslash\{i\}}} \Lambda_{g}^{\vee}(1)\right\rangle_{g, \ell} P_{m}\left(t, t_{i}\right) d t \otimes d t_{i} \otimes d \hat{\xi}_{n_{L \backslash\{i\}}}\left(t_{L \backslash\{i\}}\right) \\
& +\left(\sum_{a, b, n_{L}}\left\langle\tau_{a} \tau_{b} \tau_{n_{L}} \Lambda_{g-1}^{\vee}(1)\right\rangle_{g-1, \ell+2}\right. \\
& \left.+\sum_{\substack{g_{1}+g_{2}=g \\
I \sqcup J=L}}^{\text {stable }} \sum_{\substack{a, n_{I} \\
b, n_{J}}}\left\langle\tau_{a} \tau_{n_{I}} \Lambda_{g_{1}}^{\vee}(1)\right\rangle_{g_{1},|I|+1}\left\langle\tau_{b} \tau_{n_{J}} \Lambda_{g_{2}}^{\vee}(1)\right\rangle_{g_{2},|J|+1}\right) P_{a, b}(t) d t \otimes d \hat{\xi}_{n_{L}}\left(t_{L}\right) .
\end{aligned}
$$

Here $L=\{1, \ldots, \ell\}$ is an index set, and for a subset $I \subset L$, we denote

$$
t_{I}=\left(t_{i}\right)_{i \in I}, \quad n_{I}=\left\{n_{i} \mid i \in I\right\}, \quad \tau_{n_{I}}=\prod_{i \in I} \tau_{n_{i}}, \quad d \hat{\xi}_{n_{I}}\left(t_{I}\right)=\bigotimes_{i \in I} \frac{d}{d t_{i}} \hat{\xi}_{n_{i}}\left(t_{i}\right) d t_{i} .
$$


The last summation in the formula is taken over all partitions of $g$ and decompositions of $L$ into disjoint subsets $I \sqcup J=L$ subject to the stability condition $2 g_{1}-1+|I|>0$ and $2 g_{2}-1+|J|>0$.

Remark 5.5. An immediate observation we can make from 1.5 is the simple form of the formula for the case with one marked point:

$$
\begin{aligned}
& \sum_{n \leq 3 g-2}\left\langle\tau_{n} \Lambda_{g}^{\vee}(1)\right\rangle_{g, 1} \frac{d}{d t} \hat{\xi}_{n}(t) \\
= & \sum_{a+b \leq 3 g-4}\left(\left\langle\tau_{a} \tau_{b} \Lambda_{g-1}^{\vee}(1)\right\rangle_{g-1,2}+\sum_{g_{1}+g_{2}=g}^{\text {stable }}\left\langle\tau_{a} \Lambda_{g_{1}}^{\vee}(1)\right\rangle_{g_{1}, 1}\left\langle\tau_{b} \Lambda_{g_{2}}^{\vee}(1)\right\rangle_{g_{2}, 1}\right) P_{a, b}(t) .
\end{aligned}
$$

\section{§6. Analysis of the Laplace transforms on the Lambert curve}

As a preparation for Section 7 where we give a proof of $\sqrt{1.5}$, in this section we present analysis tools that provide the relation among the Laplace transforms on the Lambert curve 2.7). The mystery of the work of Bouchard-Mariño [3] lies in their $\zeta_{n}(y)$-forms that play an effective role in devising the topological recursion for the Hurwitz numbers. We have already identified these differential forms as polynomial forms $d \hat{\xi}_{n}(t)$, where $\hat{\xi}_{n}(t)$ 's are the Lambert $W$-function and its derivatives.

Recall Stirling's formula

$$
\begin{aligned}
\log \Gamma(z)= & \frac{1}{2} \log 2 \pi+\left(z-\frac{1}{2}\right) \log z-z \\
& +\sum_{r=1}^{m} \frac{B_{2 r}}{2 r(2 r-1)} z^{-2 r+1}-\frac{1}{2 m} \int_{0}^{\infty} \frac{B_{2 m}(x-[x])}{(z+x)^{2 m}} d x
\end{aligned}
$$

where $m$ is an arbitrary cut-off parameter, $B_{r}(s)$ is the Bernoulli polynomial defined by

$$
\frac{z e^{z x}}{e^{z}-1}=\sum_{n=0}^{\infty} B_{r}(x) \frac{z^{r}}{r !},
$$

$B_{r}=B_{r}(0)$ is the Bernoulli number, and $[x]$ is the largest integer not exceeding $x \in \mathbb{R}$. For $N>0$, we have

$$
\begin{aligned}
e^{-N} & \frac{N^{N+n}}{N !} \\
& =\frac{1}{\sqrt{2 \pi}} N^{n-1 / 2} \exp \left(-\sum_{r=1}^{m} \frac{B_{2 r}}{2 r(2 r-1)} N^{-2 r+1}\right) \exp \left(\frac{1}{2 m} \int_{0}^{\infty} \frac{B_{2 m}(x-[x])}{(N+x)^{2 m}} d x\right) .
\end{aligned}
$$


Let us define the coefficients $s_{k}$ for $k \geq 0$ by

$$
\text { (6.2) } \begin{aligned}
\sum_{k=0}^{\infty} s_{k} N^{-k} & =\exp \left(-\sum_{r=1}^{\infty} \frac{B_{2 r}}{2 r(2 r-1)} N^{-2 r+1}\right) \\
& =1-\frac{1}{12} N^{-1}+\frac{1}{288} N^{-2}+\frac{139}{51840} N^{-3}-\frac{571}{2488320} N^{-4}+\cdots .
\end{aligned}
$$

Then for a large $N$ we have an asymptotic expansion

$$
e^{-N} \frac{N^{N+n}}{N !} \sim \frac{1}{\sqrt{2 \pi}} N^{n-1 / 2} \sum_{k=0}^{\infty} s_{k} N^{-k} .
$$

Definition 6.1. Let us introduce an infinite sequence of Laurent series

$$
\eta_{n}(v)=\frac{1}{v} \sum_{k=0}^{\infty} s_{k} \frac{(2(n-k)-1) ! !}{v^{2(n-k)}}=-\eta_{n}(-v)
$$

for every $n \in \mathbb{Z}$, where $s_{k}$ 's are the coefficients defined in 6.2.

The following lemma relates the polynomial forms $\xi_{n}(t)=d \hat{\xi}_{n}(t)$, the functions $\eta_{n}(v)$, and the Laplace transform.

Proposition 6.2. For $n \geq 0$, we have

$$
\int_{0}^{\infty} e^{-s} \frac{s^{s+n}}{\Gamma(s+1)} e^{-s w} d s=\eta_{n}(v)+\text { const }+O(w)
$$

with the choice of the branch of $\sqrt{w}$ specified by $v=-\sqrt{2 w}$ as in 2.8 and 2.15, where $O(w)$ denotes a holomorphic function in $w=\frac{1}{2} v^{2}$ defined around $w=0$ which vanishes at $w=0$. The substitution of (2.15) in $\eta_{n}(v)$ yields

$$
\eta_{n}(v)=\frac{1}{2}\left(\eta_{n}(v)-\eta_{n}(-v)\right)=\frac{1}{2}\left(\hat{\xi}_{n}(t)-\hat{\xi}_{n}(s(t))\right),
$$

where $s(t)$ is the involution of 2.9. This formula is valid for $n \geq-1$, and in particular, we have a relation between the kernel and $\eta_{-1}(v)$ :

$$
\eta_{-1}(v)=\frac{1}{2}\left(\hat{\xi}_{-1}(t)-\hat{\xi}_{-1}(s(t))\right)=\frac{1}{2} \frac{t-s(t)}{t s(t)} .
$$

More precisely, for $n \geq-1$, we have

$$
\left\{\begin{array}{l}
\eta_{n}(v)=\hat{\xi}_{n}(t)+F_{n}(w), \\
\eta_{n}(-v)=\hat{\xi}_{n}(s(t))+F_{n}(w),
\end{array}\right.
$$

where $F_{n}(w)$ is a holomorphic function in $w$. 
Proof. From definition (6.4), it is obvious that the series $\eta_{n}(v)$ satisfies the recursion relation

$$
\eta_{n+1}(v)=-\frac{1}{v} \frac{d}{d v} \eta_{n}(v)
$$

for all $n \in \mathbb{Z}$. The integral 6.5 also satisfies the same recursion for $n \geq 0$. So choose an $n \geq 0$. We have an estimate

$$
e^{-s} \frac{s^{s+n}}{\Gamma(s+1)}=\frac{1}{\sqrt{2 \pi}} s^{n-1 / 2} \sum_{k=0}^{n} s_{k} s^{-k}+O\left(s^{-3 / 2}\right),
$$

valid for $s>1$. Since the integral

$$
\int_{0}^{1} e^{-s} \frac{s^{s+n}}{\Gamma(s+1)} e^{-s w} d s
$$

is an entire function in $w$, we have

$$
\begin{aligned}
& \int_{0}^{\infty} e^{-s} \frac{s^{s+n}}{\Gamma(s+1)} e^{-s w} d s=\int_{0}^{1} e^{-s} \frac{s^{s+n}}{\Gamma(s+1)} e^{-s w} d s+\int_{1}^{\infty} e^{-s} \frac{s^{s+n}}{\Gamma(s+1)} e^{-s w} d s \\
& =\int_{0}^{\infty}\left(\frac{1}{\sqrt{2 \pi}} s^{n-1 / 2} \sum_{k=0}^{n} s_{k} s^{-k}\right) e^{-s w} d s+\int_{1}^{\infty} O\left(s^{-3 / 2}\right) e^{-s w} d s+\text { const }+O(w) \\
& =\frac{1}{v} \sum_{k=0}^{n} s_{k} \frac{(2(n-k)-1) ! !}{v^{2(n-k)}}+\mathrm{const}+v O(w)+O(w) .
\end{aligned}
$$

This formula is valid for all $n \geq 0$. Starting it from a large $n \gg 0$ and using the recursion 6.9 backwards, we conclude that the $v O(w)$ terms in the above formula are indeed the positive power terms of $\eta_{n}(v)$. The principal part of $\eta_{n}(v)$ does not depend on the addition of positive power terms in $w=\frac{1}{2} v^{2}$, since $-\frac{1}{v} \frac{d}{d v}$ transforms a positive even power of $v$ to a nonnegative even power and does not create any negative powers. This proves 6.5.

Next let us estimate the holomorphic error term $O(w)$ in $(6.5)$. When $n \leq-1$, the Laplace transform 6.5 does not converge. However, the truncated integral

$$
\int_{1}^{\infty} e^{-s} \frac{s^{s+n}}{\Gamma(s+1)} e^{-s w} d s
$$

always converges and defines a holomorphic function in $v=-\sqrt{2 w}$, which still satisfies the recursion relation (6.9). Again by the inverse induction, we have

$$
\int_{1}^{\infty} e^{-s} \frac{s^{s+n}}{\Gamma(s+1)} e^{-s w} d s=\eta_{n}(v)+O(w)
$$

for every $n<0$. Now by the Euler summation formula, for $n \leq-1$ and $\operatorname{Re}(w)>0$, 
we have

$$
\begin{aligned}
\int_{1}^{\infty} & e^{-s} \frac{s^{s+n}}{\Gamma(s+1)} e^{-s w} d s-\sum_{k=2}^{\infty} e^{-k} \frac{k^{k+n}}{k !} e^{-k w} \\
& =-\frac{1}{2} e^{-(w+1)}+\int_{1}^{\infty}\left(s-[s]-\frac{1}{2}\right) \frac{d}{d s}\left(e^{-s} \frac{s^{s+n}}{\Gamma(s+1)} e^{-s w}\right) d s
\end{aligned}
$$

Note that the RHS of 6.11 is holomorphic in $w$ around $w=0$. From 2.10, 6.10 and 6.11, we establish a comparison formula

$$
\eta_{-1}(v)-\hat{\xi}_{-1}(t)=F_{-1}(w)
$$

where $F_{-1}(w)$ is a holomorphic function in $w$ defined near $w=0$, and we identify the coordinates $t, v$ and $w$ by the relations (2.16) and (2.15). Note that the relation 2.16) is invariant under the involution

$$
v \mapsto-v, \quad t \mapsto s(t) .
$$

Therefore, we also have

$$
\eta_{-1}(-v)-\hat{\xi}_{-1}(s(t))=F_{-1}(w)
$$

Thus we obtain

$$
\eta_{-1}(v)=\frac{1}{2}\left(\hat{\xi}_{-1}(t)-\hat{\xi}_{-1}(s(t))\right),
$$

which proves 6.7). Since $-\frac{1}{v} \frac{d}{d v}=t^{2}(t-1) \frac{d}{d t}$, the recursion relations 6.9 and 2.11 for $\hat{\xi}_{n}(t)$ are exactly the same. We note that from 4.12 we have

$$
-\frac{1}{v} \frac{d}{d v}=t^{2}(t-1) \frac{d}{d t}=s(t)^{2}(s(t)-1) \frac{d}{d s(t)} .
$$

Therefore, the differences $\hat{\xi}_{n+1}(t)-\hat{\xi}_{n+1}(s(t))$ satisfy the same recursion

$$
\hat{\xi}_{n+1}(t)-\hat{\xi}_{n+1}(s(t))=t^{2}(t-1) \frac{d}{d t}\left(\hat{\xi}_{n}(t)-\hat{\xi}_{n}(s(t))\right) .
$$

The recursions (6.9) and (6.14), together with the initial condition (6.7), establish 6.6. Application of the differential operator

$n+1$ times to 6.12 yields

$$
-\frac{1}{v} \frac{d}{d v}=-\frac{d}{d w}=t^{2}(t-1) \frac{d}{d t}
$$

$$
\eta_{n}(v)-\hat{\xi}_{n}(t)=F_{n}(w),
$$

where $F_{n}(w)=(-1)^{n} \frac{d^{n}}{d w^{n}} F_{-1}(w)$ is a holomorphic function in $w$ around $w=0$. Involution 6.13) then gives

$$
\eta_{n}(-v)-\hat{\xi}_{n}(s(t))=F_{n}(w) .
$$

This completes the proof of the proposition. 
As we have noted in Section 5. the residue calculations appearing in the Bouchard-Mariño recursion formula 4.16 are essentially evaluations of the product of $\xi$-forms at the points $t$ and $s(t)$ on the Lambert curve, if we truncate the result to the polynomial part. In terms of the $v$-coordinate, these two points correspond to $v$ and $-v$. Thus we have

Corollary 6.3. The residue polynomials of (5.3) are given by

$$
\begin{aligned}
& P_{a, b}(t) d t \\
& \quad=\frac{1}{2}\left[\frac{t s(t)}{t-s(t)} \frac{d t}{t^{2}(t-1)}\left(\hat{\xi}_{a+1}(t) \hat{\xi}_{b+1}(s(t))+\hat{\xi}_{a+1}(s(t)) \hat{\xi}_{b+1}(t)\right)\right]_{+} \\
& \quad=\frac{1}{2}\left[\left.\frac{\eta_{a+1}(v) \eta_{b+1}(v)}{\eta_{-1}(v)} v d v\right|_{v=v(t)}\right]_{+},
\end{aligned}
$$

where the reciprocal of

$$
\eta_{-1}(v)=\sum_{k=0}^{\infty} s_{k}(2(-1-k)-1) ! ! v^{2 k+1}=-v\left(1+\sum_{k=1}^{\infty}(-1)^{k} s_{k} \frac{1}{(2 k+1) ! !} v^{2 k}\right)
$$

is defined by

$$
\frac{1}{\eta_{-1}(v)}=-\frac{1}{v}\left(1+\sum_{m=0}^{\infty}\left(\sum_{k=1}^{\infty}(-1)^{k-1} s_{k} \frac{1}{(2 k+1) ! !} v^{2 k}\right)^{m}\right) .
$$

Proof. Using the formulas established in Proposition 6.2, we compute

$$
\begin{gathered}
\frac{1}{2} \frac{t s(t)}{t-s(t)} \frac{d t}{t^{2}(t-1)}\left(\hat{\xi}_{a+1}(t) \hat{\xi}_{b+1}(s(t))+\hat{\xi}_{a+1}(s(t)) \hat{\xi}_{b+1}(t)\right) \\
=-\frac{t s(t)}{t-s(t)} \frac{d t}{t^{2}(t-1)}\left(\frac{\hat{\xi}_{a+1}(t)-\hat{\xi}_{a+1}(s(t))}{2} \frac{\hat{\xi}_{b+1}(t)-\hat{\xi}_{b+1}(s(t))}{2}\right. \\
\left.\quad-\frac{\hat{\xi}_{a+1}(t)+\hat{\xi}_{a+1}(s(t))}{2} \frac{\hat{\xi}_{b+1}(t)+\hat{\xi}_{b+1}(s(t))}{2}\right) \\
=-\frac{1}{2 \eta_{-1}(v)}\left(\eta_{a+1}(v) \eta_{b+1}(v)-F_{a+1}(w) F_{b+1}(w)\right)(-v) d v \\
=\frac{\eta_{a+1}(v) \eta_{b+1}(v)}{2 \eta_{-1}(v)} v d v+(\text { const }+O(w)) d v .
\end{gathered}
$$

From 2.15 we see $\left[\left.(\text { const }+O(w)) d v\right|_{v \mapsto t}\right]_{+}=0$. This completes the proof of 6.15 .

For the terms involving the Cauchy differentiation kernel $B\left(t_{i}, t_{j}\right)$, we have the following formula. 
Proposition 6.4. As a polynomial in $t$ and $t_{j}$, we have the following equality:

$$
\begin{aligned}
P_{n}\left(t, t_{j}\right) d t \otimes d t_{j} & =d_{t_{j}}\left[\frac{t s(t)}{t-s(t)}\left(\frac{\hat{\xi}_{n+1}(t) d s(t)}{s(t)-t_{j}}+\frac{\hat{\xi}_{n+1}(s(t)) d t}{t-t_{j}}\right)\right]_{+} \\
& =d_{t_{j}}\left[\left.\frac{\eta_{n+1}\left(v_{j}\right)}{\eta_{-1}(v)} \cdot \frac{1}{v^{2}} \sum_{m=0}^{\text {finite }}\left(\frac{v_{j}}{v}\right)^{2 m} v d v\right|_{\substack{v=v(t) \\
v_{j}=v\left(t_{j}\right)}}\right]_{+} .
\end{aligned}
$$

On the RHS we first evaluate the expression at $v=v(t)$ and $v_{j}=v\left(t_{j}\right)$, then expand it as a series in $1 / t$ and $1 / t_{j}$, and finally truncate it as a polynomial in both $t$ and $t_{j}$.

Proof. From the formulas for $\hat{\xi}_{n}(t)$ and $\eta_{n}(v)$, we know that both expressions have the same degree $2 n+2$ in $t$ and $t_{j}$. Since the powers of $v_{j}$ in the summation $\sum_{m=0}^{\text {finite }}\left(v_{j} / v\right)^{2 m}$ are nonnegative, clearly we have

$$
d_{t_{j}}\left[\left.\frac{\eta_{n+1}(v)}{\eta_{-1}(v)} \cdot \frac{1}{v^{2}} \sum_{m=0}^{\text {finite }}\left(\frac{v_{j}}{v}\right)^{2 m} v d v\right|_{\substack{v=v(t) \\ v_{j}=v\left(t_{j}\right)}}\right]_{+}=0 .
$$

Thus we can replace the RHS of $(6.16)$ by

$$
d_{t_{j}}\left[\left.\frac{\eta_{n+1}(v)-\eta_{n+1}\left(v_{j}\right)}{\eta_{-1}(v)} \cdot \frac{1}{v^{2}} \sum_{m=0}^{\text {finite }}\left(\frac{v_{j}}{v}\right)^{2 m}(-v) d v\right|_{\substack{v=v(t) \\ v_{j}=v\left(t_{j}\right)}}\right]_{+} .
$$

Since the degree of $\eta_{n+1}\left(v\left(t_{j}\right)\right)$ in $t_{j}$ is $2 n+3$, the finite sum over $m$ in the above expression contributes nothing for $m>n+2$. Therefore,

$$
\begin{aligned}
& d_{t_{j}}\left[\left.\frac{\eta_{n+1}(v)-\eta_{n+1}\left(v_{j}\right)}{\eta_{-1}(v)} \cdot \frac{1}{v^{2}} \sum_{m=0}^{\text {finite }}\left(\frac{v_{j}}{v}\right)^{2 m}(-v) d v\right|_{\substack{v=v(t) \\
v_{j}=v\left(t_{j}\right.}}\right]_{+} \\
& =d_{t_{j}}\left[\left.\frac{t s(t)}{t-s(t)}\left(\frac{\hat{\xi}_{n+1}(t)-\hat{\xi}_{n+1}\left(t_{j}\right)}{w-w_{j}}+\frac{F_{n+1}(w)-F_{n+1}\left(w_{j}\right)}{w-w_{j}}\right)(-d w)\right|_{\substack{v=v(t) \\
v_{j}=v\left(t_{j}\right)}}\right]_{+} \\
& =d_{t_{j}}\left[\left.\frac{t s(t)}{t-s(t)} \frac{\hat{\xi}_{n+1}(t)-\hat{\xi}_{n+1}\left(t_{j}\right)}{w-w_{j}}(-d w)\right|_{\substack{v=v(t) \\
v_{j}=v\left(t_{j}\right)}}\right]_{+}
\end{aligned}
$$

because of 6.8. We also used the fact that

$$
\frac{1}{v^{2}} \sum_{m=0}^{\text {finite }}\left(\frac{v_{j}}{v}\right)^{2 m} v d v=\frac{1}{2} \frac{d w}{w-w_{j}}+O\left(w_{j}^{n+2}\right) d w
$$

and that $\frac{F_{n+1}(w)-F_{n+1}\left(w_{j}\right)}{w-w_{j}}$ is holomorphic along $w=w_{j}$. Let us use once again $-\hat{\xi}_{n+1}(t)=\hat{\xi}_{n+1}(s(t))+2 F_{n+1}(w)$ and

$$
-\frac{d w}{w-w_{j}}=-\frac{2 v d v}{v^{2}-v_{j}^{2}}=\left(\frac{1}{-v-v_{j}}-\frac{1}{v-v_{j}}\right) d v .
$$


We obtain

$$
\begin{aligned}
d_{t_{j}}\left[\left.\frac{t s(t)}{t-s(t)} \frac{\hat{\xi}_{n+1}(t)-\hat{\xi}_{n+1}\left(t_{j}\right)}{w-w_{j}}(-d w)\right|_{\substack{v=v(t) \\
v_{j}=v\left(t_{j}\right.}}\right]_{+} \\
=d_{t_{j}}\left[\left.\frac{t s(t)}{t-s(t)}\left(\frac{\hat{\xi}_{n+1}(t)-\hat{\xi}_{n+1}\left(t_{j}\right)}{-v-v_{j}} \frac{d v}{d s(t)} d s(t)\right)\right|_{\substack{v=v(t) \\
v_{j}=v\left(t_{j}\right)}}\right]_{+} \\
\quad+d_{t_{j}}\left[\left.\frac{t s(t)}{t-s(t)}\left(\frac{\hat{\xi}_{n+1}(s(t))-\hat{\xi}_{n+1}\left(s\left(t_{j}\right)\right)}{v-v_{j}} \frac{d v}{d t} d t\right)\right|_{\substack{v=v(t) \\
v_{j}=v\left(t_{j}\right)}}\right]_{+} \\
=d_{t_{j}}\left[\frac{t s(t)}{t-s(t)}\left(\frac{\hat{\xi}_{n+1}(t)-\hat{\xi}_{n+1}\left(t_{j}\right)}{s(t)-t_{j}} \frac{s(t)-t_{j}}{v(s(t))-v\left(t_{j}\right)} \frac{d v(s(t))}{d s(t)} d s(t)\right)\right]_{+} \\
\quad+d_{t_{j}}\left[\frac{t s(t)}{t-s(t)}\left(\frac{\hat{\xi}_{n+1}(s(t))-\hat{\xi}_{n+1}\left(s\left(t_{j}\right)\right)}{t-t_{j}} \frac{t-t_{j}}{v(t)-v\left(t_{j}\right)} \frac{d v(t)}{d t} d t\right)\right]_{+} .
\end{aligned}
$$

Here we remark that

$$
\begin{gathered}
d_{t_{j}}\left[\frac{t s(t)}{t-s(t)}\left(\frac{\hat{\xi}_{n+1}(t)-\hat{\xi}_{n+1}\left(t_{j}\right)}{s(t)-t_{j}} d s(t)+\frac{\hat{\xi}_{n+1}(s(t))-\hat{\xi}_{n+1}\left(s\left(t_{j}\right)\right)}{t-t_{j}} d t\right)\right]_{+} \\
=d_{t_{j}}\left[\frac{t s(t)}{t-s(t)}\left(\frac{\hat{\xi}_{n+1}(t)}{s(t)-t_{j}} d s(t)+\frac{\hat{\xi}_{n+1}(s(t))}{t-t_{j}} d t\right)\right]_{+}
\end{gathered}
$$

because the extra terms on the LHS do not contribute to the polynomial part in $t$. Therefore, it suffices to show that

$$
\begin{aligned}
& d_{t_{j}}\left[\frac{t s(t)}{t-s(t)} \frac{\hat{\xi}_{n+1}(t)-\hat{\xi}_{n+1}\left(t_{j}\right)}{s(t)-t_{j}}\left(\frac{s(t)-t_{j}}{v(s(t))-v\left(t_{j}\right)} \frac{d v(s(t))}{d s(t)}-1\right) d s(t)\right]_{+} \\
& \quad+d_{t_{j}}\left[\frac{t s(t)}{t-s(t)} \frac{\hat{\xi}_{n+1}(s(t))-\hat{\xi}_{n+1}\left(s\left(t_{j}\right)\right)}{t-t_{j}}\left(\frac{t-t_{j}}{v(t)-v\left(t_{j}\right)} \frac{d v(t)}{d t}-1\right) d t\right]_{+} \\
& =d_{t_{j}}\left[\frac{t s(t)}{t-s(t)}\left(\hat{\xi}_{n+1}(t)-\hat{\xi}_{n+1}\left(t_{j}\right)\right)\left(\frac{-d v(t)}{-v(t)-v\left(t_{j}\right)}-\frac{d s(t)}{s(t)-t_{j}}\right)\right]_{+} \\
& \quad+d_{t_{j}}\left[\frac{t s(t)}{t-s(t)}\left(\hat{\xi}_{n+1}(s(t))-\hat{\xi}_{n+1}\left(s\left(t_{j}\right)\right)\left(\frac{d v(t)}{v(t)-v\left(t_{j}\right)}-\frac{d t}{t-t_{j}}\right)\right]_{+}\right. \\
& =d_{t_{j}}\left[\frac{t s(t)}{t-s(t)}\left(\hat{\xi}_{n+1}(t)-\hat{\xi}_{n+1}\left(t_{j}\right)\right)\left(\frac{-d v(t)}{-v(t)-v\left(t_{j}\right)}-\frac{d s(t)}{s(t)-t_{j}}\right)\right]_{+} \\
& \quad-d_{t_{j}}\left[\frac{t s(t)}{t-s(t)}\left(\hat{\xi}_{n+1}(t)-\hat{\xi}_{n+1}\left(t_{j}\right)\right)\left(\frac{d v(t)}{v(t)-v\left(t_{j}\right)}-\frac{d t}{t-t_{j}}\right)\right]_{+}=0
\end{aligned}
$$

in light of 6.8 . At this stage we need the following lemma: 
Lemma 6.5. For every $n \geq 0$ we have the identity

$$
0=d_{t_{j}}\left[\left.\left(t^{n}-t_{j}^{n}\right)\left(\frac{-d v}{-v-v_{j}}-\frac{d s(t)}{s(t)-t_{j}}-\frac{d v}{v-v_{j}}+\frac{d t}{t-t_{j}}\right)\right|_{\substack{v=v(t) \\ v_{j}=v\left(t_{j}\right)}}\right]_{+} .
$$

Proof of Lemma. First let us recall that $B\left(t, t_{j}\right)=d_{t_{j}}\left(\frac{d t}{t-t_{j}}\right)$ is the Cauchy differentiation kernel of the Lambert curve $C$, which is a symmetric quadratic form on $C \times C$ with second order poles along the diagonal $t=t_{j}$. The function $v=v(t)$ is a local coordinate change, which transforms $v=0$ to $t=\infty$. Therefore, the form $\frac{d v}{v-v_{j}}-\frac{d t}{t-t_{j}}$ is a meromorphic 1-form locally defined on $C \times C$, which is actually holomorphic on a neighborhood of the diagonal and vanishes on the diagonal. Therefore, it has the Taylor series expansion in $1 / t$ and $1 / t_{j}$ without a constant term.

Since $v(s(t))=-v(t)$, the form $\frac{d v}{v+v_{j}}-\frac{d s(t)}{s(t)-t_{j}}$ is the pull-back of $\frac{d v}{v-v_{j}}-\frac{d t}{t-t_{j}}$ via the local involution $s: C \rightarrow C$ that is applied to the first factor. Thus this is again a local holomorphic 1-form on $C \times C$ and has exactly the same Taylor expansion in $1 / s(t)$ and $1 / t_{j}$. Therefore, in the $1 / t_{j}$-expansion of the difference $\frac{-d v}{-v-v_{j}}-\frac{d s(t)}{s(t)-t_{j}}-\frac{d v}{v-v_{j}}+\frac{d t}{t-t_{j}}$, no coefficient contains a constant term because it is canceled by taking the difference. This implies that the difference 1 -form does not contain any terms without $1 / t$. In other words, we have

$$
0=\left[\left.t_{j}^{n}\left(\frac{-d v}{-v-v_{j}}-\frac{d s(t)}{s(t)-t_{j}}-\frac{d v}{v-v_{j}}+\frac{d t}{t-t_{j}}\right)\right|_{\substack{v=v(t) \\ v_{j}=v\left(t_{j}\right)}}\right]_{+} .
$$

Note that we have an expression of the form

$$
\left[\frac{-d v}{-v-v_{j}}-\frac{d s(t)}{s(t)-t_{j}}-\frac{d v}{v-v_{j}}+\frac{d t}{t-t_{j}}\right]_{\substack{v=v(t) \\ v_{j}=v\left(t_{j}\right)}}=f\left(t^{-1}\right)+\frac{1}{t_{j}} F\left(\frac{1}{t}, \frac{1}{t_{j}}\right)
$$

where $f$ is a power series in one variable and $F$ a power series in two variables. Therefore,

$$
0=d_{t_{j}}\left[\left.t^{n}\left(\frac{-d v}{-v-v_{j}}-\frac{d s(t)}{s(t)-t_{j}}-\frac{d v}{v-v_{j}}+\frac{d t}{t-t_{j}}\right)\right|_{\substack{v=v(t) \\ v_{j}=v\left(t_{j}\right)}}\right]_{+} .
$$

The lemma follows from 6.19 and 6.21 .

It is obvious from 6.19 and 6.20 that

$$
0=d_{t_{j}}\left[\left.\left(t^{n}-t_{j}^{n}\right) \frac{t s(t)}{t-s(t)}\left(\frac{-d v}{-v-v_{j}}-\frac{d s(t)}{s(t)-t_{j}}-\frac{d v}{v-v_{j}}+\frac{d t}{t-t_{j}}\right)\right|_{\substack{v=v(t) \\ v_{j}=v\left(t_{j}\right)}}\right]_{+}
$$

Since $\hat{\xi}_{n+1}(t)$ is a polynomial in $t, 6.17$ follows from 6.22 . This completes the proof of the proposition. 


\section{$\S 7$. Proof of the Bouchard-Mariño topological recursion formula}

In this section we prove 1.5 . Since it is equivalent to Conjecture 4.8, we establish the Bouchard-Mariño conjecture. Our procedure is to take the direct image of the equation 3.12 on the Lambert curve via the projection $\pi: C \rightarrow \mathbb{C}$. To compute the direct image, it is easier to switch to the coordinate $v$ of the Lambert curve, because of the relation 2.17). This simple relation tells us that the direct image of a function $f(v)$ on $C$ via the projection $\pi: C \rightarrow \mathbb{C}$ is just the even powers of the $v$-variable in $f(v)$ :

$$
\pi_{*} f=f(v)+f(-v) .
$$

After taking the direct image, we extract the principal part of the meromorphic function in $v$, which becomes the Bouchard-Mariño recursion 1.5. To this end, we utilize the formulas developed in Section 6 .

Here again let us consider the $\ell=1$ case first. We start with Proposition 3.4 .

Theorem 7.1. We have the equality

$$
\begin{gathered}
-\sum_{n \leq 3 g-2}\left\langle\tau_{n} \Lambda_{g}^{\vee}(1)\right\rangle_{g, 1} \eta_{-1}(v) \eta_{n+1}(v)=\frac{1}{2} \sum_{a+b \leq 3 g-4}\left[\left\langle\tau_{a} \tau_{b} \Lambda_{g-1}^{\vee}(1)\right\rangle_{g-1,2}\right. \\
\left.+\sum_{g_{1}+g_{2}=g}^{\text {stable }}\left\langle\tau_{a} \Lambda_{g_{1}}^{\vee}(1)\right\rangle_{g_{1}, 1}\left\langle\tau_{b} \Lambda_{g_{2}}^{\vee}(1)\right\rangle_{g_{2}, 1}\right]\left(\eta_{a+1}(v) \eta_{b+1}(v)+O_{w}(1)\right),
\end{gathered}
$$

where $O_{w}(1)$ denotes a holomorphic function in $w=\frac{1}{2} v^{2}$.

Proof. We use 6.8 to change from the $t$-variables to the $v$-variables. The function factor of the LHS of (3.6) becomes

$$
(2 g-1) \eta_{n}(v)+\eta_{n+1}(v)-\eta_{-1}(v) \eta_{n+1}(v)+v f_{L}(w)+\text { const }+O(w),
$$

where $f_{L}(w)$ is a Laurent series in $w$. The function factor of the RHS is

$$
\eta_{a+1}(v) \eta_{b+1}(v)+v f_{R}(w)+\text { const }+O(w),
$$

where $f_{R}(w)$ is another Laurent series in $w$. We note that the product of two $\eta_{n^{-}}$ functions is a Laurent series in $w$. Therefore, extracting the principal part of the Laurent series in $w$, we obtain

$$
\begin{aligned}
- & \sum_{n \leq 3 g-2}\left\langle\tau_{n} \Lambda_{g}^{\vee}(1)\right\rangle_{g, 1} \eta_{-1}(v) \eta_{n+1}(v)=\frac{1}{2} \sum_{a+b \leq 3 g-4}\left[\left\langle\tau_{a} \tau_{b} \Lambda_{g-1}^{\vee}(1)\right\rangle_{g-1,2}\right. \\
& \left.+\sum_{g_{1}+g_{2}=g}^{\text {stable }}\left\langle\tau_{a} \Lambda_{g_{1}}^{\vee}(1)\right\rangle_{g_{1}, 1}\left\langle\tau_{b} \Lambda_{g_{2}}^{\vee}(1)\right\rangle_{g_{2}, 1}\right]\left(\eta_{a+1}(v) \eta_{b+1}(v)+\text { const }+O(w)\right),
\end{aligned}
$$

which completes the proof of the theorem. 
Corollary 7.2. The cut-and-join equation (3.5) for the case of $\ell=1$ implies the topological recursion 5.6 .

Proof. Going back to the $t$-coordinates and using $(2.10), 2.11$, and 6.8 in (7.1), we establish

$$
\begin{aligned}
& \sum_{n \leq 3 g-2}\left\langle\tau_{n} \Lambda_{g}^{\vee}(1)\right\rangle_{g, 1} \xi_{n}(t)=\frac{1}{2} \sum_{a+b \leq 3 g-4}\left(\left\langle\tau_{a} \tau_{b} \Lambda_{g-1}^{\vee}(1)\right\rangle_{g-1,2}\right. \\
& \left.\quad+\sum_{g_{1}+g_{2}=g}^{\text {stable }}\left\langle\tau_{a} \Lambda_{g_{1}}^{\vee}(1)\right\rangle_{g_{1}, 1}\left\langle\tau_{b} \Lambda_{g_{2}}^{\vee}(1)\right\rangle_{g_{2}, 1}\right)\left[\left.\frac{\eta_{a+1}(v) \eta_{b+1}(v)}{\eta_{-1}(v)} v d v\right|_{v=v(t)}\right]_{+},
\end{aligned}
$$

since

$$
\left[\left.\frac{\text { const }+O(w)}{\eta_{-1}(v)} v d v\right|_{v=v(t)}\right]_{+}=0 .
$$

From Corollary 6.3, we conclude that 7.2 is identical to 5.6 . This completes the proof of the topological recursion for $\ell=1$.

We are now ready to give a proof of 1.5 . The starting point is the Laplace transform of the cut-and-join equation, as established in Theorem 3.7. Since we are interested in the principal part of the formula in the $v$-coordinate expansion, in what follows we ignore all terms that contain any positive powers of $v_{i}$ 's.

First let us deal with the unstable $(0,2)$-terms computed in (3.9). Using (6.9), we find

$$
-\frac{\partial}{\partial w_{i}} \widehat{\mathcal{H}}_{0,2}\left(t_{i}, t_{j}\right) \equiv-\frac{1}{v_{i}} \frac{\partial}{\partial v_{i}} \log \left(\eta_{-1}\left(v_{i}\right)-\eta_{-1}\left(v_{j}\right)\right) \equiv \frac{\eta_{0}\left(v_{i}\right)}{\eta_{-1}\left(v_{i}\right)-\eta_{-1}\left(v_{j}\right)}
$$

modulo holomorphic functions in $w_{i}$ and $w_{j}$. Therefore, the result of the coordinate change from the $t$-coordinates to the $v$-coordinates is the following:

$$
\begin{gathered}
\sum_{n_{L}}\left\langle\tau_{n_{L}} \Lambda_{g}^{\vee}(1)\right\rangle_{g, \ell}\left((2 g-2+\ell) \eta_{n_{L}}\left(v_{L}\right)+\sum_{i=1}^{\ell} \eta_{n_{i}+1}\left(v_{i}\right) \eta_{L \backslash\{i\}}\left(v_{L \backslash\{i\}}\right)\right. \\
\left.-\sum_{i=1}^{\ell} \eta_{-1}\left(v_{i}\right) \eta_{n_{i}+1}\left(v_{i}\right) \eta_{n_{L \backslash\{i\}}}\left(v_{L \backslash\{i\}}\right)\right) \\
\equiv \frac{1}{2} \sum_{i=1}^{\ell} \sum_{n_{L \backslash\{i\}}} \sum_{a, b}\left(\left\langle\tau_{a} \tau_{b} \tau_{n_{L \backslash\{i\}}} \Lambda_{g-1}^{\vee}(1)\right\rangle_{g-1, \ell+1}\right. \\
\left.+\sum_{\substack{g_{1}+g_{2}=g \\
I \sqcup J=L \backslash\{i\}}}^{\operatorname{stable}}\left\langle\tau_{a} \tau_{n_{I}} \Lambda_{g_{1}}^{\vee}(1)\right\rangle_{g_{1},|I|+1}\left\langle\tau_{b} \tau_{n_{J}} \Lambda_{g_{2}}^{\vee}(1)\right\rangle_{g_{2},|J|+1}\right) \\
\times \eta_{a+1}\left(v_{i}\right) \eta_{b+1}\left(v_{i}\right) \eta_{n_{L \backslash\{i\}}}\left(v_{L \backslash\{i\}}\right)
\end{gathered}
$$




$$
\begin{aligned}
+\frac{1}{2} \sum_{i=1}^{\ell} \sum_{j \neq i} \sum_{n_{L \backslash\{i, j\}}} \sum_{m}\left\langle\tau_{n_{L \backslash\{i, j\}}} \tau_{m} \Lambda_{g}^{\vee}(1)\right\rangle_{g, \ell-1} \eta_{n_{L \backslash\{i, j\}}}\left(v_{L \backslash\{i, j\}}\right) \\
\times \frac{\eta_{m+1}\left(v_{i}\right) \eta_{0}\left(v_{i}\right)-\eta_{m+1}\left(v_{j}\right) \eta_{0}\left(v_{j}\right)}{\eta_{-1}\left(v_{i}\right)-\eta_{-1}\left(v_{j}\right)},
\end{aligned}
$$

again modulo terms containing any holomorphic terms in any $w_{k}$. At this stage we take the direct image with respect to the projection $\pi: C \rightarrow \mathbb{C}$ applied to the $v_{1^{-}}$ coordinate component, and then restrict the result to its principal part, meaning that we throw away any terms that contain nonnegative powers of any $v_{k}$. Thanks to (6.8), only those terms containing $\eta_{a}\left(v_{1}\right) \eta_{b}\left(v_{1}\right)$ survive. The last term of 7.3 . requires a separate care. We find

$$
\begin{gathered}
\frac{1}{2}\left(\frac{\eta_{m+1}\left(v_{1}\right) \eta_{0}\left(v_{1}\right)-}{\eta_{-1}\left(v_{1}\right)-} \eta_{m+1}\left(v_{j}\right) \eta_{0}\left(v_{j}\right)\right. \\
\left.=-\left(\eta_{m+1}\left(v_{1}\right) \eta_{0}\left(v_{1}\right)-\eta_{m+1}\left(v_{j}\right) \eta_{0}\left(v_{j}\right)\right) \frac{\eta_{m+1}\left(v_{1}\right) \eta_{0}\left(v_{1}\right)-\eta_{m+1}\left(v_{j}\right) \eta_{0}\left(v_{j}\right)}{\eta_{-1}\left(v_{1}\right)^{2}-\eta_{-1}\left(v_{j}\right)^{2}}\right) \\
\equiv \frac{\eta_{m+1}\left(v_{j}\right)}{v_{1}^{2}-v_{j}^{2}}=\frac{\eta_{m+1}\left(v_{j}\right)}{v_{1}^{2}} \sum_{k=0}^{\infty}\left(\frac{v_{j}}{v_{1}}\right)^{2 k},
\end{gathered}
$$

modulo terms containing nonnegative terms in $v_{j}$. Thus by taking the direct image and reducing to the principal part, 7.3 is greatly simplified. We have obtained:

Theorem 7.3. Modulo terms with holomorphic factors in $v_{k}$,

$$
\begin{aligned}
& -\sum_{n_{L}}\left\langle\tau_{n_{L}} \Lambda_{g}^{\vee}(1)\right\rangle_{g, \ell} \eta_{-1}\left(v_{1}\right) \eta_{n_{1}+1}\left(v_{1}\right) \eta_{n_{L \backslash\{1\}}}\left(v_{L \backslash\{1\}}\right) \\
& \equiv \frac{1}{2} \sum_{n_{L \backslash\{1\}}} \sum_{a, b}\left(\left\langle\tau_{a} \tau_{b} \tau_{n_{L \backslash\{1\}}} \Lambda_{g-1}^{\vee}(1)\right\rangle_{g-1, \ell+1}\right. \\
& \left.+\sum_{\substack{g_{1}+g_{2}=g \\
I \sqcup J=L \backslash\{1\}}}^{\text {stable }}\left\langle\tau_{a} \tau_{n_{I}} \Lambda_{g_{1}}^{\vee}(1)\right\rangle_{g_{1},|I|+1}\left\langle\tau_{b} \tau_{n_{J}} \Lambda_{g_{2}}^{\vee}(1)\right\rangle_{g_{2},|J|+1}\right) \\
& \times \eta_{a+1}\left(v_{1}\right) \eta_{b+1}\left(v_{1}\right) \eta_{n_{L \backslash\{1\}}}\left(v_{L \backslash\{1\}}\right) \\
& +\frac{1}{2} \sum_{j \geq 1} \sum_{n_{L \backslash\{1, j\}}} \sum_{m}\left\langle\tau_{n_{L \backslash\{1, j\}}} \tau_{m} \Lambda_{g}^{\vee}(1)\right\rangle_{g, \ell-1} \eta_{n_{L \backslash\{1, j\}}}\left(v_{L \backslash\{1, j\}}\right) \\
& \times \frac{\eta_{m+1}\left(v_{j}\right)}{v_{1}^{2}} \sum_{k=0}^{\text {finite }}\left(\frac{v_{j}}{v_{1}}\right)^{2 k} .
\end{aligned}
$$

We note that only finitely many terms of the expansion contribute in the last term of (7.4). Appealing to Corollary 6.3 and Proposition 6.4. we obtain (1.5), after switching back to the $t$-coordinates. We have thus completed the proof of the Bouchard-Mariño conjecture [3]. 
Appendix. Examples of linear Hodge integrals and Hurwitz numbers

In this Appendix we give a few examples of linear Hodge integrals and Hurwitz numbers computed by Michael Reinhard.

Table 1. Examples of linear Hodge integrals.

\begin{tabular}{|c|c|c|c|c|c|c|}
\hline \multicolumn{7}{|c|}{$g=2$} \\
\hline$\ell=1$ & $\left\langle\tau_{3} \lambda_{1}\right\rangle$ & $\frac{1}{480}$ & & & & \\
\hline$\ell=2$ & $\left\langle\tau_{2}^{2} \lambda_{1}\right\rangle$ & $\frac{5}{576}$ & & & & \\
\hline \multicolumn{7}{|c|}{$g=3$} \\
\hline$\ell=1$ & $\left\langle\tau_{6} \lambda_{1}\right\rangle$ & $\frac{7}{138240}$ & $\left\langle\tau_{5} \lambda_{2}\right\rangle$ & $\frac{41}{580608}$ & & \\
\hline \multirow{2}{*}{$\ell=2$} & $\left\langle\tau_{2} \tau_{5} \lambda_{1}\right\rangle$ & $\frac{323}{483840}$ & $\left\langle\tau_{2} \tau_{4} \lambda_{2}\right\rangle$ & $\frac{2329}{2903040}$ & & \\
\hline & $\left\langle\tau_{3} \tau_{4} \lambda_{1}\right\rangle$ & $\frac{19}{17920}$ & $\left\langle\tau_{3}^{2} \lambda_{2}\right\rangle$ & $\frac{1501}{1451520}$ & & \\
\hline$\ell=3$ & $\left\langle\tau_{2}^{2} \tau_{4} \lambda_{1}\right\rangle$ & $\frac{541}{60480}$ & $\left\langle\tau_{2} \tau_{3}^{3} \lambda_{1}\right\rangle$ & $\frac{89}{7680}$ & $\left\langle\tau_{2}^{2} \tau_{3} \lambda_{2}\right\rangle$ & $\frac{859}{96768}$ \\
\hline$\ell=4$ & $\left\langle\tau_{2}^{3} \tau_{3} \lambda_{1}\right\rangle$ & $\frac{395}{3456}$ & $\left\langle\tau_{2}^{4} \lambda_{2}\right\rangle$ & $\frac{17}{192}$ & & \\
\hline \multicolumn{7}{|c|}{$g=4$} \\
\hline$\ell=1$ & $\left\langle\tau_{9} \lambda_{1}\right\rangle$ & $\frac{1}{1244160}$ & $\left\langle\tau_{8} \lambda_{2}\right\rangle$ & $\frac{1357}{696729600}$ & $\left\langle\tau_{7} \lambda_{3}\right\rangle$ & $\frac{13}{6220800}$ \\
\hline \multirow{4}{*}{$\ell=2$} & $\left\langle\tau_{2} \tau_{8} \lambda_{1}\right\rangle$ & $\frac{841}{38707200}$ & $\left\langle\tau_{2} \tau_{7} \lambda_{2}\right\rangle$ & $\frac{33391}{696729600}$ & $\left\langle\tau_{3} \tau_{5} \lambda_{3}\right\rangle$ & $\frac{2609}{29030400}$ \\
\hline & $\left\langle\tau_{3} \tau_{7} \lambda_{1}\right\rangle$ & $\frac{221}{4147200}$ & $\left\langle\tau_{3} \tau_{6} \lambda_{2}\right\rangle$ & $\frac{1153}{11059200}$ & $\left\langle\tau_{4}^{2} \lambda_{3}\right\rangle$ & $\frac{6421}{58060800}$ \\
\hline & $\left\langle\tau_{4} \tau_{6} \lambda_{1}\right\rangle$ & $\frac{517}{5806080}$ & $\left\langle\tau_{4} \tau_{5} \lambda_{2}\right\rangle$ & $\frac{979}{6451200}$ & & \\
\hline & $\left\langle\tau_{5}^{2} \lambda_{1}\right\rangle$ & $\frac{1223}{11612160}$ & $\left\langle\tau_{2} \tau_{6} \lambda_{3}\right\rangle$ & $\frac{5477}{116121600}$ & & \\
\hline \multirow{4}{*}{$\ell=3$} & $\left\langle\tau_{2}^{2} \tau_{7} \lambda_{1}\right\rangle$ & $\frac{3487}{5806080}$ & $\left\langle\tau_{3} \tau_{4}^{2} \lambda_{1}\right\rangle$ & $\frac{137}{46080}$ & $\left\langle\tau_{3}^{2} \tau_{4} \lambda_{2}\right\rangle$ & $\frac{58951}{16588800}$ \\
\hline & $\left\langle\tau_{2} \tau_{3} \tau_{6} \lambda_{1}\right\rangle$ & $\frac{50243}{38707200}$ & $\left\langle\tau_{2}^{2} \tau_{6} \lambda_{2}\right\rangle$ & $\frac{137843}{116121600}$ & $\left\langle\tau_{2}^{2} \tau_{5} \lambda_{3}\right\rangle$ & $\frac{241}{230400}$ \\
\hline & $\left\langle\tau_{2} \tau_{4} \tau_{5} \lambda_{1}\right\rangle$ & $\frac{2597}{1382400}$ & $\left\langle\tau_{2} \tau_{3} \tau_{5} \lambda_{2}\right\rangle$ & $\frac{577}{258048}$ & $\left\langle\tau_{2} \tau_{3} \tau_{4} \lambda_{3}\right\rangle$ & $\frac{27821}{16588800}$ \\
\hline & $\left\langle\tau_{3}^{2} \tau_{5} \lambda_{1}\right\rangle$ & $\frac{3359}{1382400}$ & $\left\langle\tau_{2} \tau_{4}^{2} \lambda_{2}\right\rangle$ & $\frac{2657}{967680}$ & $\left\langle\tau_{3}^{3} \lambda_{3}\right\rangle$ & $\frac{4531}{2073600}$ \\
\hline \multicolumn{7}{|c|}{$g=5$} \\
\hline \multirow{2}{*}{$\ell=1$} & $\left\langle\tau_{12} \lambda_{1}\right\rangle$ & $\frac{1}{106168320}$ & $\left\langle\tau_{10} \lambda_{3}\right\rangle$ & $\frac{71}{1114767360}$ & & \\
\hline & $\left\langle\tau_{11} \lambda_{2}\right\rangle$ & $\frac{577}{16721510400}$ & $\left\langle\tau_{9} \lambda_{4}\right\rangle$ & $\frac{21481}{367873228800}$ & & \\
\hline
\end{tabular}


Some examples of $g=5$ Hurwitz numbers:

$$
\begin{array}{ll}
h_{5,(1)}=0, & h_{5,(4)}=272097280 \\
h_{5,(2)}=1 / 2, & h_{5,(5)}=333251953125 \\
h_{5,(3)}=59049, & h_{5,(6)}=202252053177720 .
\end{array}
$$

\begin{tabular}{|c|c|c|c|c|}
\hline$h_{g, \mu}$ & $g=1$ & $g=2$ & $g=3$ & $g=4$ \\
\hline (1) & 0 & 0 & 0 & 0 \\
\hline$(2)$ & $1 / 2$ & $1 / 2$ & $1 / 2$ & $1 / 2$ \\
\hline$(1,1)$ & $1 / 2$ & $1 / 2$ & $1 / 2$ & $1 / 2$ \\
\hline (3) & 9 & 81 & 729 & 6561 \\
\hline$(2,1)$ & 40 & 364 & 3280 & 29524 \\
\hline$(1,1,1)$ & 40 & 364 & 3280 & 29524 \\
\hline (4) & 160 & 5824 & 209920 & 7558144 \\
\hline$(3,1)$ & 1215 & 45927 & 1673055 & 60407127 \\
\hline$(2,2)$ & 480 & 17472 & 629760 & 22674432 \\
\hline$(2,1,1)$ & 5460 & 206640 & 7528620 & 271831560 \\
\hline$(1,1,1,1)$ & 5460 & 206640 & 7528620 & \\
\hline (5) & 3125 & 328125 & 33203125 & 3330078125 \\
\hline$(4,1)$ & 35840 & 3956736 & 409108480 & 41394569216 \\
\hline$(3,2)$ & 26460 & 2748816 & 277118820 & 27762350616 \\
\hline$(3,1,1)$ & 234360 & 26184060 & 2719617120 & 275661886500 \\
\hline$(2,2,1)$ & 188160 & 20160000 & 2059960320 & 207505858560 \\
\hline$(2,1,1,1)$ & 1189440 & 131670000 & 13626893280 & \\
\hline$(1,1,1,1,1)$ & 1189440 & 131670000 & & \\
\hline (6) & 68040 & 16901136 & 3931876080 & 895132294056 \\
\hline$(5,1)$ & 1093750 & 287109375 & 68750000000 & 15885009765625 \\
\hline$(4,2)$ & 788480 & 192783360 & 44490434560 & 10093234511360 \\
\hline$(4,1,1)$ & 9838080 & 2638056960 & 638265788160 & 148222087453440 \\
\hline$(3,3)$ & 357210 & 86113125 & 19797948720 & 4487187539835 \\
\hline$(3,2,1)$ & 14696640 & 3710765520 & 872470478880 & 199914163328880 \\
\hline$(3,1,1,1)$ & 65998800 & 17634743280 & 4259736280800 & \\
\hline$(2,2,2)$ & 2016000 & 486541440 & 111644332800 & 25269270586560 \\
\hline$(2,2,1,1)$ & 80438400 & 20589085440 & 4874762692800 & \\
\hline$(2,1,1,1,1)$ & 382536000 & 100557737280 & & \\
\hline$(1,1,1,1,1,1)$ & 382536000 & & & \\
\hline
\end{tabular}

Table 2. Examples of Hurwitz numbers for $1 \leq g \leq 4$ and $|\mu| \leq 6$. 


\section{Acknowledgements}

Our special thanks are due to Vincent Bouchard for numerous discussions and tireless explanations of the recursion formulas and the remodeling theory. We thank the Institute for the Physics and Mathematics of the Universe, the Osaka City University Advanced Mathematical Institute, and the American Institute of Mathematics for their hospitality during our stays and for providing us with the opportunity of collaboration. Without their assistance, this collaboration would have never been started. We also thank Gaëtan Borot, Yon-Seo Kim, Chiu-Chu Melissa Liu, Kefeng Liu, Marcos Mariño, Nicolas Orantin, and Hao Xu for discussions, and Michael Reinhard for his permission to reproduce his computation of linear Hodge integrals and Hurwitz numbers in this paper.

During the period of preparation of this work, B.E.'s research was supported in part by the ANR project Grandes Matrices Aléatoires ANR-08-BLAN-0311-01, the European Science Foundation through the Misgam program, and the Quebec government with the FQRNT; M.M. received financial support from the NSF, Kyoto University, Tôhoku University, KIAS in Seoul, and the University of Salamanca; and B.S. received support from IPMU.

\section{References}

[1] G. Borot, B. Eynard, M. Mulase and B. Safnuk, Hurwitz numbers, matrix models and topological recursion, J. Geom. Phys. (2011), to appear.

[2] V. Bouchard, A. Klemm, M. Mariño and S. Pasquetti, Remodeling the B-model, Comm. Math. Phys. 287 (2008), 117-178. Zbl 1178.81214 MR 2480744

[3] V. Bouchard and M. Mariño, Hurwitz numbers, matrix models and enumerative geometry, in From Hodge theory to integrability and TQFT tt*-geometry, Proc. Sympos. Pure Math. 78, Amer. Math. Soc., 2008, 263-283. Zbl 1151.14335 MR 2483754

[4] L. Chen, Y. Li and K. Liu, Localization, Hurwitz numbers and the Witten conjecture, Asian J. Math. 12 (2008), 511-518. Zbl pre05554939 MR 2481688

[5] R. Dijkgraaf, Intersection theory, integrable hierarchies and topological field theory, in: New symmetry principles in quantum field theory (Cargèse, 1991), NATO Adv. Sci. Inst. Ser. B Phys. 295, Plenum, New York, 1992, 95-158. MR 1204453

[6] R. Dijkgraaf and C. Vafa, Two dimensional Kodaira-Spencer theory and three dimensional Chern-Simons gravity, arXiv:0711.1932 [hep-th].

[7] R. Dijkgraaf, E. Verlinde and H. Verlinde, Loop equations and Virasoro constraints in non-perturbative two-dimensional quantum gravity, Nucl. Phys. B348 (1991), 435-456. MR 1083914

[8] T. Ekedahl, S. Lando, M. Shapiro and A. Vainshtein, Hurwitz numbers and intersections on moduli spaces of curves, Invent. Math. 146 (2001), 297-327. Zbl 1073.14041 MR 1864018

[9] B. Eynard, Topological expansion for the 1-hermitian matrix model correlation functions, J. High Energy Phys. 2004, no. 11, 031, 35 pp. MR 2118807

[10] $\_$, Recursion between volumes of moduli spaces, arXiv:0706.4403 [math-ph]. 
[11] _ All order asymptotic expansion of large partitions, J. Statist. Mech. Theory Exp. 2008, no. 7, P07023, 34 pp. MR 2439683

[12] B. Eynard, M. Mariño and N. Orantin, Holomorphic anomaly and matrix models, J. High Energy Phys. 2007, no. 6, 058, 20 pp. MR 2326593

[13] B. Eynard and N. Orantin, Invariants of algebraic curves and topological expansion, Comm. Number Theory Phys. 1 (2007), 347-452. Zbl 1161.14026

[14] Weil-Petersson volume of moduli spaces, Mirzakhani's recursion and matrix models, arXiv:0705.3600 [math-ph].

[15] C. Faber and R. Pandharipande, Hodge integrals and Gromov-Witten theory, Invent. Math. 139 (2000), 173-199. Zbl 0960.14031 MR 1728879

[16] Hodge integrals, partition matrices, and the $\lambda_{g}$ conjecture, Ann. of Math. 157 (2003), 97-124. Zbl 1058.14046 MR 1954265

[17] I. P. Goulden and D. M. Jackson, Transitive factorisations into transpositions and holomorphic mappings on the sphere, Proc. Amer. Math. Soc. 125 (1997), 51-60. Zbl 0861.05006 MR 1396978

[18] I. P. Goulden, D. M. Jackson and A. Vainshtein, The number of ramified coverings of the sphere by the torus and surfaces of higher genera, Ann. Combin. 4 (2000), 27-46. Zbl 0957.58011 MR 1763948

[19] I. P. Goulden, D. M. Jackson and R. Vakil, The Gromov-Witten potential of a point, Hurwitz numbers, and Hodge integrals, Proc. London Math. Soc. 83 (2001), 563-581. Zbl 1074.14520 MR 1851082

[20] A short proof of the $\lambda_{g}$-conjecture without Gromov-Witten theory: Hurwitz theory and the moduli of curves, J. Reine Angew. Math. 637 (2009), 175-191. Zbl 1205.14068 MR 2599085

[21] T. Graber and R. Vakil, Hodge integrals and Hurwitz numbers via virtual localization, Compos. Math. 135 (2003), 25-36. Zbl 1063.14032 MR 1955162

[22] _ Relative virtual localization and vanishing of tautological classes on moduli spaces of curves, Duke Math. J. 130 (2005), 1-37. Zbl 1088.14007 MR 2176546

[23] A. Hurwitz, Ueber Riemann'sche Flächen mit gegebenen Verzweigungspunkten, Math. Ann. 39 (1891), 1-60. JFM 23.0429.01 MR 1510692

[24] M. Kazarian, KP hierarchy for Hodge integrals, Adv. Math. 221 (2009), 1-21. Zbl 1168.14006 MR 2509319

[25] M. Kazarian and S. Lando, An algebro-geometric proof of Witten's conjecture, J. Amer. Math. Soc. 20 (2007), 1079-1089. Zbl 1155.14004 MR 2328716

[26] Y. S. Kim and K. Liu, A simple proof of Witten conjecture through localization, arXiv:math/0508384 [math.AG] (2005).

[27] M. Kontsevich, Intersection theory on the moduli space of curves and the matrix Airy function, Comm. Math. Phys. 147 (1992), 1-23. Zbl 0756.35081 MR 1171758

[28] A. M. Li, G. Zhao and Q. Zheng, The number of ramified coverings of a Riemann surface by Riemann surface, Comm. Math. Phys. 213 (2000), 685-696. Zbl 0973.14029||MR 1785434

[29] C.-C. M. Liu, Formulae of one-partition and two-partition Hodge integrals, Geom. Topol. Monogr. 8 (2006), 105-128. Zbl 1115.14050 MR 2402822

[30] C.-C. M. Liu, K. Liu and J. Zhou, A proof of a conjecture of Mariño-Vafa on Hodge integrals, J. Differential Geom. 65 (2003), 289-340. Zbl 1077.14084 MR 2058264

[31] K. Liu and H. Xu, A simple proof of Mirzakhani's recursion formula of Weil-Petersson volumes, arXiv:0705.2086 [math.AG].

[32] M. Mariño, Chern-Simons theory, matrix models, and topological strings, Oxford Univ. Press, 2005. Zbl 1093.81002 MR 2168778 
[33] _ Open string amplitudes and large order behavior in topological string theory, J. High Energy Phys. 2008, no. 3, 060, 34 pp. MR 2391060

[34] A. Mironov and A. Morozov, Virasoro constraints for Kontsevich-Hurwitz partition function, arXiv:0807.2843.

[35] M. Mirzakhani, Simple geodesics and Weil-Petersson volumes of moduli spaces of bordered Riemann surfaces, Invent. Math. 167 (2007), 179-222. Zbl 1125.30039 MR 2264808

[36] Weil-Petersson volumes and intersection theory on the moduli space of curves, J. Amer. Math. Soc. 20 (2007), 1-23. Zbl 1120.32008 MR 2257394

[37] M. Mulase and B. Safnuk, Mirzakhani's recursion relations, Virasoro constraints and the KdV hierarchy, Indian J. Math. 50 (2008), 189-228. Zbl 1144.14030 MR 2379144

[38] M. Mulase and N. Zhang, Polynomial recursion formula for linear Hodge integrals, Comm. Number Theory Phys. 4 (2010), 267-294. MR 2725053

[39] A. Okounkov, Random matrices and random permutations, Int. Math. Res. Notices 2000, no. 20, 1043-1095. Zbl 1018.15020 MR 1802530

[40] Toda equations for Hurwitz numbers, Math. Res. Lett. 7 (2000), 447-453. Zbl 0969.37033 MR 1783622

[41] A. Okounkov and R. Pandharipande, Gromov-Witten theory, Hurwitz numbers, and matrix models, I, in Algebraic geometry - Seattle 2005, Part 1, Proc. Sympos. Pure Math. 80, Amer. Math. Soc., 2009, 325-414. Zbl 1205.14072 MR 2483941

[42] - The equivariant Gromov-Witten theory of $\mathbb{P}^{1}$, Ann. of Math. 163 (2006), 561-605. Zbl 1105.14077 MR 2199226

[43] R. Vakil, Harvard Thesis, 1997.

[44] E. Witten, Two dimensional gravity and intersection theory on moduli space, Surveys Differential Geom. 1 (1991), 243-310. Zbl 0757.53049 MR 1144529

[45] J. Zhou, Hodge integrals, Hurwitz numbers, and symmetric groups, math.AG/0308024. 